\title{
3D biosensors in advanced medical diagnostics of high mortality diseases
}

\author{
Rita Rebelo $^{\mathrm{a}, \mathrm{b}, 1}$, Ana I. Barbosa ${ }^{\mathrm{a}, \mathrm{b}, 1}$, David Caballero ${ }^{\mathrm{a}, \mathrm{b}}$, Il Keun Kwon ${ }^{\mathrm{c}}$, Joaquim M. Oliveira ${ }^{\mathrm{a}, \mathrm{b}, \mathrm{d}}$, \\ Subhas C. Kundu ${ }^{\mathrm{a}, \mathrm{b}}$, Rui L. Reis ${ }^{\mathrm{a}, \mathrm{b}, \mathrm{c}, \mathrm{d}}$, Vitor M. Correlo ${ }^{\mathrm{a}, \mathrm{b}, \mathrm{d}, *}$ \\ a $3 B$ 's Research Group, I3Bs - Research Institute on Biomaterials, Biodegradables and Biomimetics of University of Minho, Headquarters of the European Institute of \\ Excellence on Tissue Engineering and Regenerative Medicine, AvePark-Parque de Ciência e Tecnologia, Zona Industrial da Gandra, $4805-017$ Barco, Guimarães, Portugal \\ ${ }^{\mathrm{b}}$ ICVS/3B's-PT Government Associated Laboratory, Braga, Guimarães, Portugal \\ ${ }^{\mathrm{c}}$ Department of Dental Materials, School of Dentistry, Kyung Hee University, 26, Kyungheedae-ro, Dongdaemun-gu, Seoul 02477, Republic of Korea \\ ${ }^{\mathrm{d}}$ The Discoveries Centre for Regenerative and Precision Medicine, Headquarters at University of Minho, Avepark, $4805-017$ Barco, Guimarães, Portugal
}

\section{A R T I C L E I N F O}

\section{Keywords:}

3D biosensors

Cancer

Cardiovascular diseases

Diabetes

Implantable

Organ-on-chip

\begin{abstract}
A B S T R A C T
Cardiovascular diseases, cancer, and diabetes are high mortality diseases, which account for almost two thirds of all deaths worldwide. Their early detection and continuous evaluation are fundamental for an improved patient prognosis and reduced socioeconomic impact. Current biosensor technologies are typically based on the analysis of whole blood samples from patients for the detection of disease-specific biomarkers. However, these technologies display serious shortcomings, such as reduced sensitivity and dynamic range, limited in vivo applicability, and lack of continuous monitoring. There is the urgent need for new diagnostic and treatment follow-up tools, which allow for the early detection of the pathology as well as for the continuous monitoring of the physiological responses to specific therapies. During the last years, a new generation of biosensor technologies with improved performance has emerged in the biomedical sector. The combination of advanced biomaterial methods, biochemical tools, and micro/nanotechnology approaches has resulted in the development of innovative three-dimensional (3D) biosensor platforms for advanced medical diagnosis. In this review, we report the most recent advances in the field of 3D biosensors for clinical applications, focusing on the diagnosis and monitoring of cardiovascular diseases, cancer, and diabetes. We discuss about their clinical performance compared to standard biosensor technologies, their implantable capability, and their integration into microfluidic devices to develop clinically-relevant models. Overall, we anticipate that 3D biosensors will drive us toward a new paradigm in medical diagnosis, resulting in real-time in vivo biosensors capable to significantly improve patient prognosis.
\end{abstract}

\section{Introduction}

According to the World Health Organization, ischemic heart diseases, stroke and other cardiovascular diseases (CVDs), were responsible for 17.7 million deaths worldwide in 2015. Similarly, cancer and diabetes caused 8.8 and 1.6 million deaths, respectively. All combined, these high mortality diseases killed 28.1 million people, corresponding to approximately $50 \%$ of the global deaths (WHO, 2017).

Current diagnostic assays used in the clinics for monitoring CVDs, cancer and diabetes rely on biomarker quantitation in physiological fluids, imaging, electrical signals monitoring, and tissue biopsy. These technologies are typically expensive, time consuming and performed in different health facilities, which oblige the patients to wait a long time between analysis and results. Frequently, these techniques also lack the needed sensitivity and specificity, which makes the results meaningless and creates the need to repeat the entire process. More importantly, the current diagnostic procedures lack the ability to continuously evaluate the physiological condition of a patient and their response to therapeutic treatments, limiting early pathology detection and continuous treatment follow up (Ahmed et al., 2014). Altogether, there is an urge for novel diagnostic and analytical technologies, i.e. biosensors, which are closely connected to the patient physiology and its unique

\footnotetext{
* Corresponding author at: 3B's Research Group, I3Bs - Research Institute on Biomaterials, Biodegradables and Biomimetics of University of Minho, Headquarters of

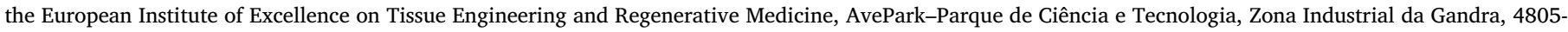
017 Barco, Guimarães, Portugal.

E-mail addresses: rita.rebelo@i3bs.uminho.pt (R. Rebelo), anaibarbosa@i3bs.uminho.pt (A.I. Barbosa), dcaballero@i3bs.uminho.pt (D. Caballero), kwoni@khu.ac.kr (I.K. Kwon), miguel.oliveira@i3bs.uminho.pt (J.M. Oliveira), kundu@i3bs.uminho.pt (S.C. Kundu), rgreis@i3bs.uminho.pt (R.L. Reis), vitorcorrelo@i3bs.uminho.pt (V.M. Correlo).

${ }^{1}$ Authors contributed equally.
} 
metabolic responses. These new generation of devices could be either implanted into the human body, allowing continuous and real-time monitoring of disease biomarkers (Muskovich and Bettinger, 2012), or integrated into an in vitro model (static or dynamic) of a patient disease to assess the efficiency or toxicity of personalized treatments.

Currently, the major studies in medical biosensors rely on miniaturized two-dimensional (2D) surfaces with immobilized biorecognition molecules. However, planar surfaces often present poor analytical performance, such as narrow dynamic range, instable immobilized probes and low limit of detection (LoD). These limitations are a direct consequence of the reduced surface area and surface area-to-volume ratio, which constrains the amount and efficient capture of a specific analyte and the signal transduction. Indeed, Barbosa and Reis recently identified the surface area and surface area-to volume ratio as significantly relevant for sensitive detection of protein biomarkers in point-of-care microfluidic tests (Barbosa and Reis, 2017). Moreover, the response time of $2 \mathrm{D}$ biosensors is typically larger, in particular for lower LoD, since the analyte relies on diffusion for transportation to the immobilized probe, which sits in a single surface. Furthermore, the effect of shear stress in a planar surface, due to sample analyte loading, can remove or inhibit the bond between the analyte and the specific immobilized probe, with significant impact in analytical performance (Barbosa, 2015). Importantly, 2D biosensors are typically non-flexible, which aggravates the foreign body response when implanted inside the human body. Finally, they typically do not allow real-time monitoring of biomarkers in the three-dimensional (3D) extracellular matrix of human tissues and organs (Bertok et al., 2014; Edmondson et al., 2014; Zhu et al., 2015).

The limitations of planar sensing platforms led to the development of miniaturized 3D biosensors. This new paradigm in biosensing technologies ally high analytical performance, biocompatibility and 3D architecture, fundamental for their integration into implantable devices and in vitro analytical models. In this review, we define 3D biosensors as those biosensing devices where the biorecognition element is adhered onto a 3D architecture platform (Fig. 1). These support materials

A

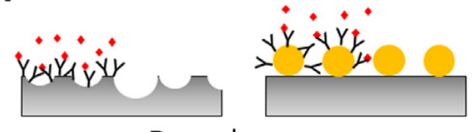

Roughness

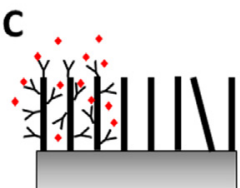

Nanotubes
D

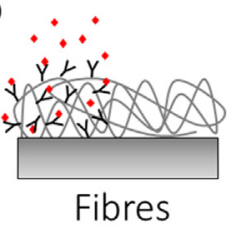

Fibres

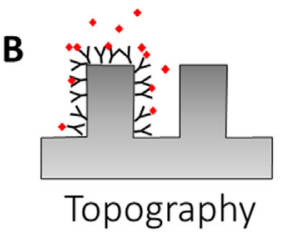

E

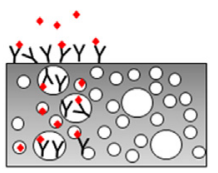

Porosity
$\mathbf{F}$

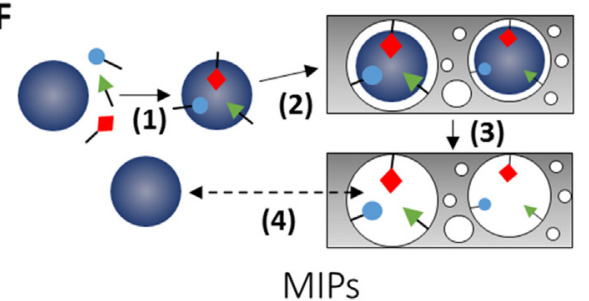

Fig. 1. Architectures typically used in 3D biosensors. A) Enhanced surface roughness; B) Topographical micro/nano-structures; C) Nanotubes; D) Fibers meshwork; E) Porous materials, and F) Molecular Imprinted Polymers (Key: 1. Self-assembly; 2. Polymerization; 3. Extraction, and 4. Rebinding). frequently display enhanced roughness, porosity, or 3D topographic features. The deposition of vertically-aligned nanotubes, 3D fibers network, or molecular imprinted polymers (MIPs) also fit within this category. These engineered 3D structures enhance the electrode area, and therefore, increase the biorecognition signal. These structures may vary from the nanometer to the mesoscopic scale, and may be fabricated by a diverse variety of techniques, including standard UV-photolithography, wet/dry etching, 3D bioprinting, or photopolymerization, among other methods (Kassing, 2006; Merceron and Murphy, 2015; Mironi-Harpaz et al., 2012).

The 3D structure of biosensors significantly impacts on the amount of immobilized biorecognition probes, and as a consequence, on the analyte capture. It also influences the electrode reaction kinetics, diffusion time, and the shear stress between the analyte and the immobilized specific probe, among other parameters, which translates into an improved analytical performance (Barbosa, 2015; Barbosa and Reis, 2017; Faghri et al., 2010). Finally, 3D structures can be easily combined with biocompatible and flexible materials, making them especially interesting for implantable applications.

Overall, 3D biosensors show high potential to revolutionize the field of medical diagnostics. Herein, we review the state-of-the-art of 3D biosensors, and present a critical evaluation of their performance for the in vitro and in vivo diagnostics of CVDs, cancer, and diabetes. To this aim, we discuss about the most common materials used as building blocks, their fabrication methods, the immobilization procedures, and the transducers typically employed. We also describe about their integration into organ-on-chip (OC) platforms and in implantable devices for advanced clinical applications. Finally, we comment on their current market opportunities and how can 3D biosensors be employed to overcome the limitations of planar sensing technologies to be adopted by the clinics.

\section{Materials and fabrication strategies}

The selection of the material and fabrication methodology is fundamental in the development of 3D biosensors, since it will affect all the other components and directly influence their performance. The selected material must synergize both with the biorecognition elements and the antigen of interest, as well as with the signal transducer (Colombo et al., 2015; Frascella et al., 2016; Guenther, 2010; L. Li et al., 2015; Qu et al., 2014; Rong et al., 2015; Slaughter and Sunday, 2014). A diverse variety of materials displaying different properties can be used for the development of 3D biosensors, including carbon, glass, silicon, metals, and polymers, among others. The choice of the adequate material depends on multiple parameters, such as the type and properties of the biosensor, the antigen of interest, and the final application (see Table 1) (Ming et al., 2016; Zhang et al., 2000).

Typically, hydrogels and/or polymers are used for the fabrication of 3D biosensors because they provide a native-like environment for maintaining the activity of the biorecognition elements, and are compatible with 3D micro/nano-fabrication techniques (Derkus et al., 2015). This also makes 3D biosensors amenable for being implanted within the human body with minimal immune response, or integrated into in vitro disease models (Jin and Dijkstra, 2010; Loh and Choong, 2013). Moreover, the use of conductive polymers leads to the fabrication of conductive hydrogels providing electrical conductivity. This allows, for instance, the entrapment of oxidoreductases and the electron transfer, enhancing the performance of glucose biosensors for diabetes monitoring (Ming et al., 2016; Zhang et al., 2000).

In the last decade, several methods for the fabrication of 3D hydrogels have been reported, including freeze-dryer, layer-by-layer, 3D bioprinting, and photo-polymerization methods (Lu and Li, 2013). However, hydrogels can also be synthesized through standard chemical means. These include one-step techniques, like crosslinking of multifunctional monomers and polymerization, and multiple-step techniques, including the synthesis of polymer molecules and its crosslinking. 
Table 1

Main advantages and disadvantages of materials for 3D biosensor applications.

\begin{tabular}{|c|c|c|}
\hline Material & Advantages & Disadvantages \\
\hline Carbon-based & $\begin{array}{l}\text { - High conductivity } \\
\text { - Low electrical resistivity; } \\
\text { - Good mechanical properties; } \\
\text { - Easy to functionalize. }\end{array}$ & $\begin{array}{l}\times \text { Non- biodegradable; } \\
\times \text { Limited data on tolerance by healthy tissues. }\end{array}$ \\
\hline Glass/Silicon & $\begin{array}{l}\text { - Biocompatible; } \\
\text { - Good optical and mechanical properties; } \\
\text { - Cost-effectiveness. }\end{array}$ & $\times$ Non- biodegradable. \\
\hline Metals & $\begin{array}{l}\text { - Good electrical and mechanical properties; } \\
\text { - Easy to functionalize. }\end{array}$ & $\begin{array}{l}\times \text { Lower biocompatibility (compared to polymers); } \\
\times \text { Non- biodegradable. }\end{array}$ \\
\hline Polymers & $\begin{array}{l}\text { - Great biocompatibility; } \\
\text { - Able to mimic the natural cell environment; } \\
\text { - Can be conductive. }\end{array}$ & $\times$ Poorer mechanical properties (compared to metals). \\
\hline
\end{tabular}

A) $\underline{O D}$

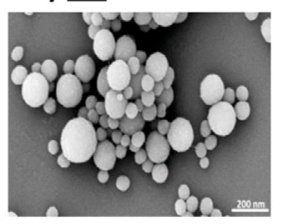

Nanoparticles

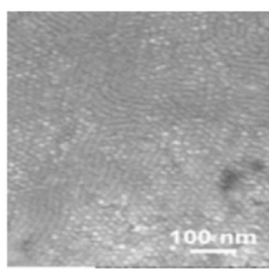

Quantum dots
B) $\underline{1 D}$

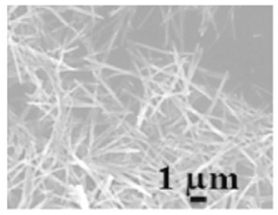

Nanowires

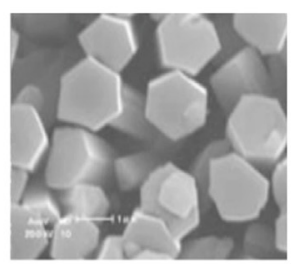

Nanoroads

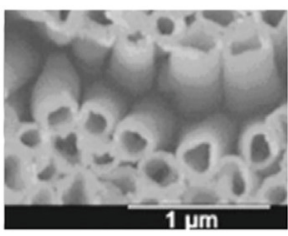

Nanotubes
C) $\underline{2 \mathrm{D}}$

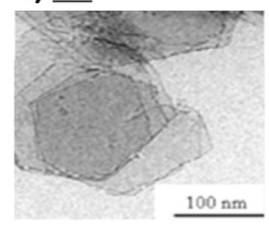

Nanosheets

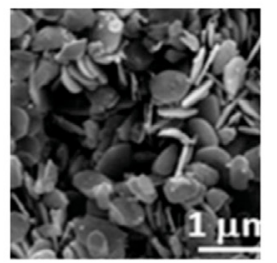

Nanoplates
D) $\underline{3 D}$

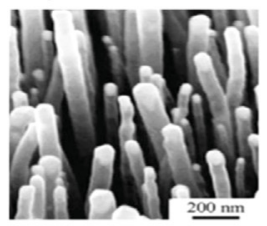

Nanopillars

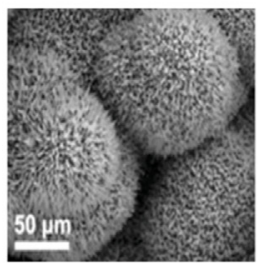

Nanoflowers

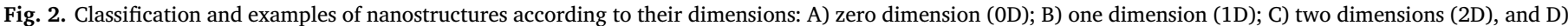
three dimensions (3D) (Tiwari et al., 2012). Figures reprinted with permission from Elsevier.

The synthesis process plays an important role, since it can tailor the final hydrogel properties, such as biodegradation, mechanical strength and chemical and biological response to stimuli (Ahmed, 2015; Akhtar et al., 2016; Berti et al., 2017; Srisuk et al., 2018).

A large plethora of micro- and nano-sized structures are employed in 3D biosensors (Fig. 2). These structures enable a closer interaction with the target molecules and increase the surface/volume ratio, which drastically improves the electric device performance (Hou et al., 2016; Malekzad et al., 2017; Zhang et al., 2000). The integration of 3D micro/ nano-structures on biosensors can be obtained by different means, including:

(i) In situ fabrication by physical/chemical etching. Typically, etching techniques such as focused ion beam, oxygen plasma, e-beam, laser-based lithography, metal-assisted chemical etching, dealloying, or standard hydrofluoric acid etching are used, among many others. Examples of micro/nano-structures manufactured by these methods include nanoporous metals (gold, alumina, silicon, silicon oxide, etc.), pillars, tips, cavities, pits, grooves, and others. As an example, dealloying was recently used to manufacture a 3D gold nanoporous electrode and applied for the development of a novel bioelectrode-based biosensor for the direct detection of sulfide, an environmental pollutant (Liu et al., 2017). The bioelectrode contained recombinant $E$. coli expressing sulfide:Quinone oxidoreductase deposited on top of the nanoporous gold electrode for sulfide detection. The results showed that the remarkable catalytic oxidation properties of the $E$. coli - 3D gold nanoporous electrode for sulfide contributed to an improved performance when compared with standard methodologies, and importantly, when tested in wastewater.

(ii) Incorporation into a previously existing $3 D$ network forming composite materials. These materials are very common in biosensors since each material retains its original properties. The resulting composite displays improved physical, chemical and mechanical characteristics of those exhibited by each individual material (Borole et al., 2012; Zhang et al., 2000). Recently, a glucose biosensor based on hollow porous $\mathrm{Co}_{3} \mathrm{O}_{4}$ nanododecahedras attached to a 3D carbon nanotube mesh was developed (S. Wang et al., 2018). This composite showed a remarkable catalytic activity towards glucose oxidation and an excellent analytical performance, 
which paves the way for the development of high-performance electrochemical non-enzymatic glucose biosensors.

(iii) Deposition/growth on a flat electrode surface. Typically, a bottom-up approach is followed using chemical/physical vapor deposition, layer-by-layer, inert gas condensation, micelle and inverse micelle methods, aerosol techniques, sol-gel, electrodeposition, or precipitation techniques for the deposition/growth of e.g., nanoparticles, anodically-grown $\mathrm{TiO}_{2}$ nanotube arrays, verticallyaligned-single and multi-walled-carbon nanotubes arrays, 3Dgrown graphene, as well as their nanocomposites (Cassell et al., 2004; Khalaf et al., 2012; Manawi et al., 2018; Rajput, 2015). For instance, Singh et al. developed a highly porous 3D nanostructure for glucose monitoring formed by carbon nanotubes and nanodiamonds deposited on the electrodes through layer-by-layer technique. The spatially controlled deposition of these nanostructures was optimized in order to obtain a porous nanostructure to allow the diffusion of biomolecules of sizes up to 3-10 nm. Next, an ultrathin polypyrene film was deposited around the 3D structure for the immobilization of the bioreceptor. The resulting 3D nanostructure improved drastically the biosensor performance when compared to the equivalent pure nanotube setup (Singh et al., 2013).

Typically, hydrogels are used for the fabrication of composite materials due to their intrinsic mechanochemical properties. In this regard, we next describe about their use for the development of 3D biosensors, highlighting their advantages for specific clinical applications.

\subsection{Hydrogels}

The intrinsic properties of hydrogels make them ideal candidates for the development of 3D biosensors. Their extremely high water content facilitates the diffusion of a larger amount of analyte towards the immobilized probes (da Silva et al., 2014; Urban and Weiss, 2009). The composition of the hydrogel also plays an important role in the biosensor performance. As an example, Colombo et al. developed a sandwich-type biosensor for glucose quantification studying the influence of hydrogel composition on the biosensor response. To this aim, glucose oxidase $\left(\mathrm{GO}_{\mathrm{X}}\right)$ was mixed with mucin and albumin and then crosslinked with glutaraldehyde (GA), resulting in the formation of a hydrogel trapped between two membranes of polycarbonate. The results showed that these membranes helped to control the diffusion and to reject eventual interferences. Further, the sensitivity of the $\mathrm{GO}_{\mathrm{X}}$ sensor was low when the GA concentration was lower than $3 \%$ and when only mucin was used in matrix preparation. The opposite behavior was observed for higher amounts of GA (Colombo et al., 2015). Similarly, Qu et al. developed a hydrogel from ferrocene, an artificial electron mediator, modified with amino acid phenylalanine for the detection of $\mathrm{GO}_{\mathrm{X}}$ via rapid self-assembly mechanism (Fig. 3A). The electron transfer between the enzyme and the electrode should be taken into account, since the redox centers of the enzymes are usually deeply embedded in the thick protein shell. Thus, the introduction of electron mediators, like ferrocene, facilitate the electron transfer. Hydrogels prepared through this technique are characterized by its easy preparation process and functionalization flexibility. Additionally, the resulting hydrogel presented a simpler molecular structure when compared with other hydrogels prepared from peptide and amino acids. The hydrogel biocompatibility helped to maintain the $\mathrm{GO}_{\mathrm{x}}$ bioactivity and the large number of ferrocene moieties acted as a mediator to $\mathrm{GO}_{\mathrm{x}}$. The use of ferrocene provided a higher sensitivity to the 3D biosensor, in contrast to glucose biosensors based on oxygen (Qu et al., 2014).

Variations in oxygen concentration are shown to influence $\mathrm{GO}_{\mathrm{x}}-$ based biosensor performance either in amperometric enzyme electrodes or in glucose-sensitive hydrogel (GSH) biosensors. However, the biosensor performance can be independent of the blood oxygen level if the GSH is enzyme-free. For that, phenylboronic acid (PBA) side chains are typically incorporated into hydrogels and, in the presence of glucose, these polymers modify their swelling properties by ionization or by the formation of glucose-mediated reversible crosslinks. Thus, an increase on glucose concentration promotes binding leading to a higher hydrophobicity and gel swelling. The transference of solvent and solute into the hydrogel can be controlled by a rigid porous membrane. This way, combining a "smart" hydrogel and a microfabricated pressure sensor in a piezoresistive biochemical sensor it is possible to build a biocompatible free-enzyme glucose biosensor (Guenther, 2010).

\subsection{Nanocomposites structures}

The integration of nanostructures, such as nanoparticles (NPs), into hydrogels can significantly enhance the performance of biosensors due to their superior electrochemical behavior and large active surface area (Kazemi et al., 2016). As an example, Rong et al. (2015) developed a conducting polypyrrole (PPy)-based hydrogel/AuNPs (gold nanoparticles) 3D electrode for the detection of CEA, a cancer biomarker. The high porosity of the PPy hydrogel was achieved mixing the PPy with phytic acid as crosslinker, and ammonium persulfate as initiator. AuNPs were electrochemically deposited on the PPy-modified electrodes resulting in a 3D nanostructured system. The electrical deposition was crucial for the dispersion of the AuNPs, which acted as an immobilization matrix and provided an electrically continuous 3D path for efficient charge collection. Similarly, Li et al. developed a 3D biosensor based on a platinum nanoparticles (PtNPs) polyaniline hydrogel electrode for the simultaneous early detection of metabolites associated to cardiovascular risk, such as uric acid, cholesterol, and triglyceride (Fig. 3B). The use of PtNPs promoted the effective electrochemical oxidization in the enzymatic reaction, and the enhancement of the current collection in overall electrochemical processes (L. Li et al., 2015).

Other nanocomposite materials, besides hydrogels, with 3D architecture were also employed for the development of 3D biosensors. Sabury et al. reported the fabrication of a 3D glucose biosensor through the immobilization of oxidase on partially reduced graphene-gold nanocomposite sheets (PRGO-AuNPs/GOx). Despite the well-known advantages of graphene, it is reported that the chemically reduced graphene oxide (RGO) may suffer restacking due to van der Waals and $\pi \mathrm{i}-\pi \mathrm{i}$ interactions, decreasing the surface area and making the processability difficult. To prevent agglomeration of graphene sheets in dry state, authors added AuNPs as biocompatible 3D nanostructures (Fig. 3C). The suitable conditions for graphene reduction were used to maintain the appropriated conductivity and keep some of the useful graphene oxide functionalities to ensure communication of immobilized enzyme with the electrode surface. The functional groups helped to achieve a stable colloid and provide homogeneous mixing of AuNPs and PRGO without any macromolecular additive. Furthermore, AuNPs enhanced the electron transfer behavior of the GOx, decreasing the detection limit and increasing the sensitivity of the biosensor (Sabury et al., 2015).

Finally, silver-based nano-pinetree arrays and carbon nanotubes were recently used to develop 3D biosensing devices for the diagnostic of acute myocardial infarction (Fig. 3D) (El-Said et al., 2016; GomesFilho et al., 2013). Despite the good results obtained in vitro, the wellknown cytotoxicity of silver and carbon nanotubes makes the applicability unrealistic for clinical applications (Arora et al., 2014; Darne et al., 2014; Knetsch and Koole, 2011; Patlolla et al., 2010; Rai et al., 2009). Nevertheless, biocompatible coatings may be used to minimize this problem, although this solution may influence the biomarker immobilization and signal transduction, altering the biosensor performance.

\section{Probe immobilization}

Biosensors for clinical applications must detect a specific analyte in 
A

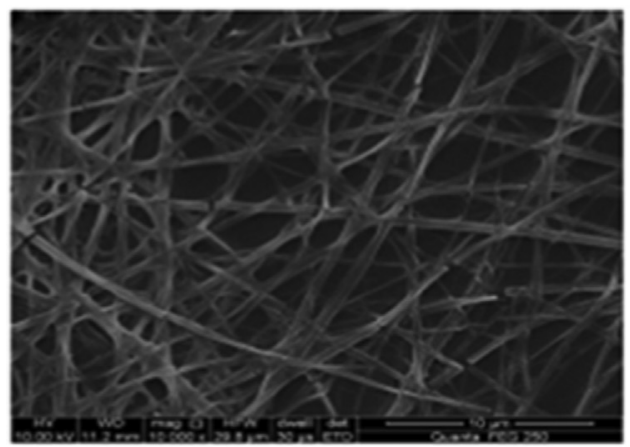

C

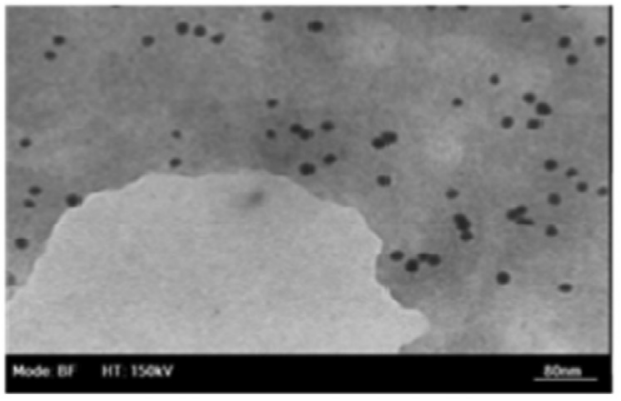

B

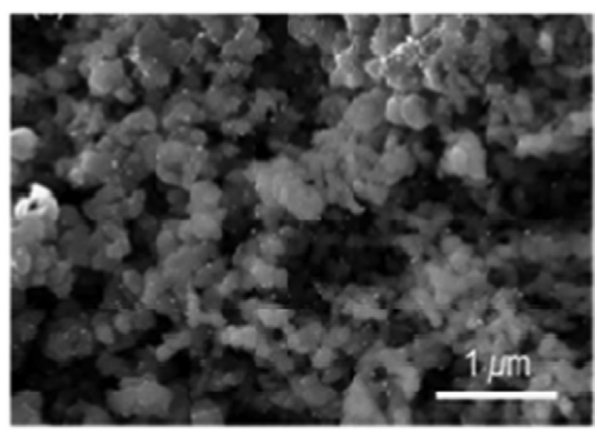

D

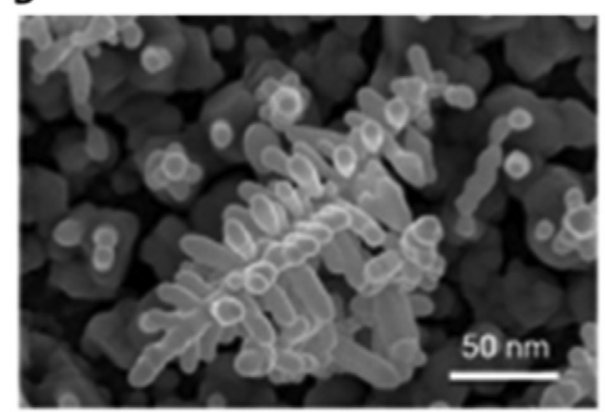

Fig. 3. Nanocomposites for the fabrication of 3D biosensors. A) SEM image of a hydrogel prepared from ferrocene modified with amino acid phenylalanine (Qu et al., 2014). B) SEM image of PtNPs loaded onto the PAni hydrogel matrix (L. Li et al., 2015). C) TEM image of AuNPs distribution into PRGO-AuNP nanocomposite (Sabury et al., 2015). D) SEM image of Ag nano-pinetree/ITO substrate (El-Said et al., 2016). Figures reproduced with permission from ACS publications and Elsevier. complex physiological fluids. For this, biorecognition probes specific to the analyte of interest need to be immobilized onto a solid support surface. The amount of captured analyte is intrinsically linked to the analytical performance of the biosensor, and will depend on the amount, the affinity, and the activity of the immobilized probes. These parameters are greatly influenced by the topography of the biosensor support material (Welch et al., 2017), and the chemical immobilization technique used (Yu et al., 2015). A 3D biosensor substrate will allow a larger amount of immobilized biorecognition probes, since it offers increased surface area when compared with planar surfaces, which are usually associated with low immobilization efficiency (Gupta and Chaudhury, 2007; Tanaka et al., 2009; Wang and Feng, 2015). In addition, 3D material supports can also improve the maintenance the biological activity of protein probes by minimizing steric hindrance, providing improved probe orientation and stability for enhanced analyte capture. This is a direct consequence of not only their high surface area, but also due to their biocompatibility, permeability and high water content (Homaei et al., 2013; Kress et al., 2002; Wang and Feng, 2015).

Even though several types of biorecognition probes can be used (Chambers et al., 2008; Martinkova et al., 2017), here we mainly focus in the immobilization of enzymes and antibodies, since glucose (diabetes) and proteins (CVDs and cancer) are the most used biomarkers for their diagnosis and monitoring. It may be noted that for diabetes, autoantibodies may also be employed as biomarkers due to their high predictive value. However, their use in the development of biosensors is still scarce in particular when compared to glucose (Taplin and Barker, 2008). Finally, other protein-based biorecognition probes, such as peptides (Etayash et al., 2015; H. Li et al., 2015), polymer brushes (J. Wang et al., 2018), antibody fragments (Saerens et al., 2008), or membrane proteins (Misawa et al., 2018), have also been employed for CVDs and cancer.

The state-of-the-art of probe immobilization reveals that the chemical immobilization techniques used in 3D environments in the last 10 years are very similar to the ones used on planar biosensors. However, the overall analytical performance of 3D biosensors is significantly improved due to the influence of the 3D architecture in the amount, biological activity, and stability of the immobilized probes.

\subsection{Enzyme immobilization}

Typically, enzymes are immobilized through their entrapment into hydrogel pores. This method allows the flow of the analyte of interest through the 3D hydrogel structure and the immobilization of the enzyme, avoiding its denaturation since the enzyme is not necessarily bound to the polymer (Mohamad et al., 2015). As an example, Qu et al. developed a glucose sensor using simple entrapment of enzymes within an hydrogel by mixing GOx during the hydrogel formation process. The developed sensor displayed a linear range between 0.1 and $20 \mathrm{mM}$, with a LoD of $50 \mu \mathrm{M}$, making this 3D biosensor clinically relevant for the diagnostics of diabetes mellitus (Qu et al., 2014) (Fig. 4A). The major drawback of enzyme entrapment is the mass transfer limitation of the analyte to the enzyme active site. Also, enzyme leakage may occur if the pores of the support matrix are too large, as well as deactivation during immobilization, low loading capacity and abrasion of support material during usage (Górecka and Jastrzębska, 2011). New strategies have been reported for improving enzyme entrapment in glucose biosensors to improve their performance. For example, GOx enzymes were first cross-linked and entrapped in a polyethylene glycol hydrogel in a micropatterned glucose biosensor. However, since the expected biosensor performance was not achieved, further studies were performed with covalently bond GOx enzymes to modified AuNPs using mercaptoundecanoic acid and citrate-stabilized (Fig. 4B). The conjugated AuNPs were then entrapped inside a micropatterned hydrogel (Pedrosa et al., 2011). Interestingly, this approach allowed a 100-fold increment on the sensitivity of the sensor. Another approach for improving enzyme entrapment was developed by Bornhoeft et al., who reported a 3D biosensor where $\mathrm{GO}_{\mathrm{x}}$ was encapsulated inside alginate microparticles embedded in polyelectrolyte multilayer. The microparticles were embedded in alginate hydrogel with a glucose fluorescent indicator, creating an alginate-in-alginate response material. The biosensor response was given by an immobilized phosphorescent porphyrin dye inside the alginate microparticles, whose phosphorescence lifetimes could be correlated with glucose concentration (Bornhoeft et al., 2017). 
A
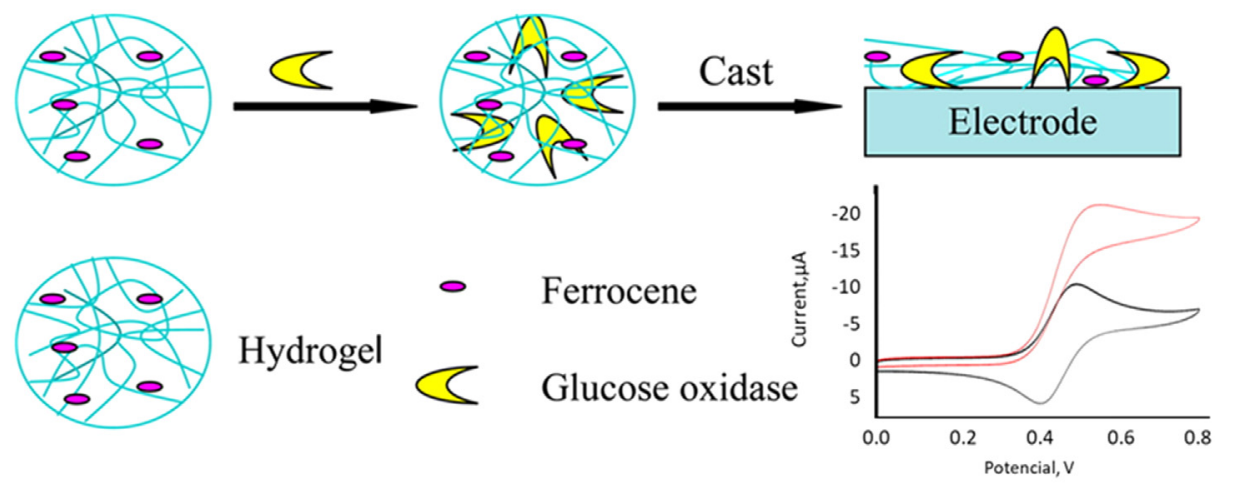

B

a)

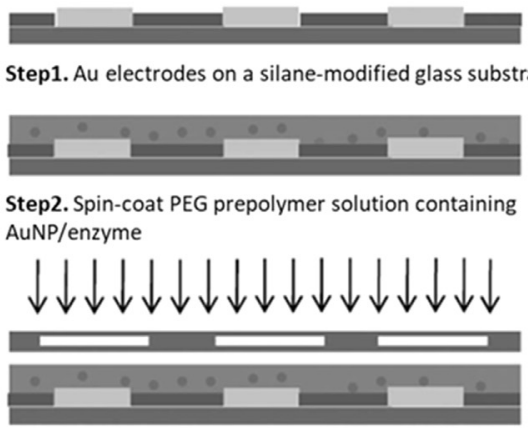

Step3. Expos to UV through photomask

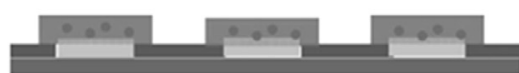

Step4. Wash in DI water to remove the non-polymerized PEG

b)

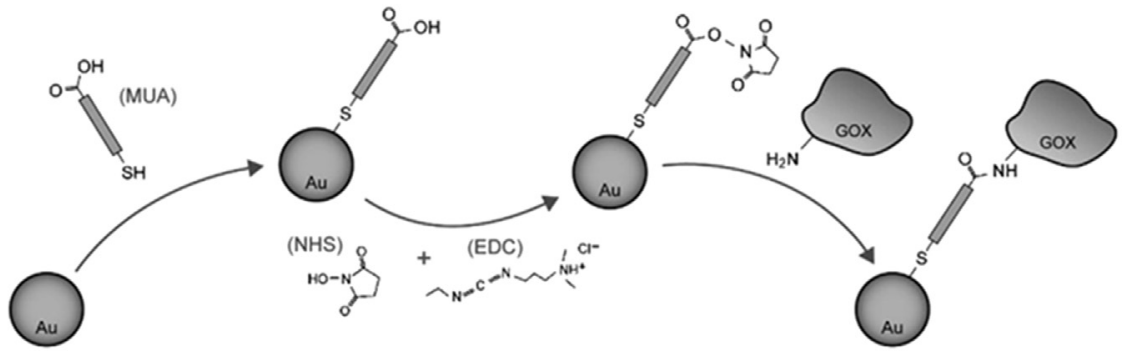

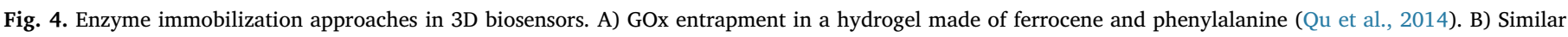

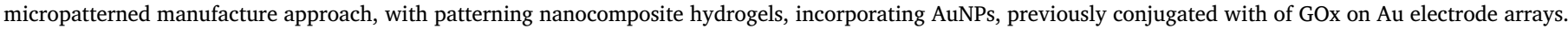

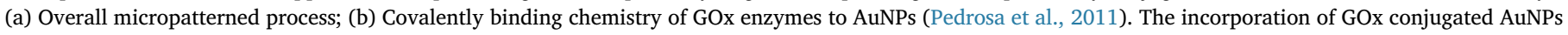
allowed 100-fold improvement on glucose sensitivity. Figures reproduced with permission from ACS publications and Wiley.

\subsection{Antibody immobilization}

Antibodies are globular proteins with specific molecular regions for antigen binding. Unlike enzymes, antigen-antibody reaction is affinitybased, therefore stoichiometric in nature. Consequently, the finite surface area in a biosensor, and the lack of biological activity, has an increased negative impact in the analytical performance of biosensors compared to the enzymatic ones. 3D surface topographies can increase the amount of immobilized antibody and their biological activity due to an increment in the total surface area available and antibody molecular stability, and decrease in denaturation and steric hindrance effects (Rogers, 2000; Wang and Feng, 2015; Welch et al., 2017).

For instance, Chen et al. reported the development of a 3D biosensor by adding 11-mercaptoundecanoic and acid/6-mercapto-1-hexanol
(MUA/MCH) monolayer onto a gold surface. This monolayer was followed by highly branched dextran amine (DA), which allowed the formation of a 3D architecture. Finally, acetylated (HA-7) molecules were added to the branched DA, ensuring the orientation and activity of the immobilized antibody by affinity binding (Fig. 5A). The characterization of the 3D topography was performed by atomic force microscopy, which showed an increase in roughness from 3 to $8 \mathrm{~nm}$ when dextran-amine was added to the modified surface (Fig. 5B). To emphasize the importance of the 3D topography, antibodies were immobilized directly on the MUA/MCH monolayer, and then, onto DA and HA-7 peptides. Electrochemical impedance spectroscopy measurements showed a higher capacity for antibody immobilization on the 3D scaffold, in contrast to MUA/MCH monolayer (Fig. 5C) (Chen et al., 2014). This enhanced antibody immobilization, together with their 


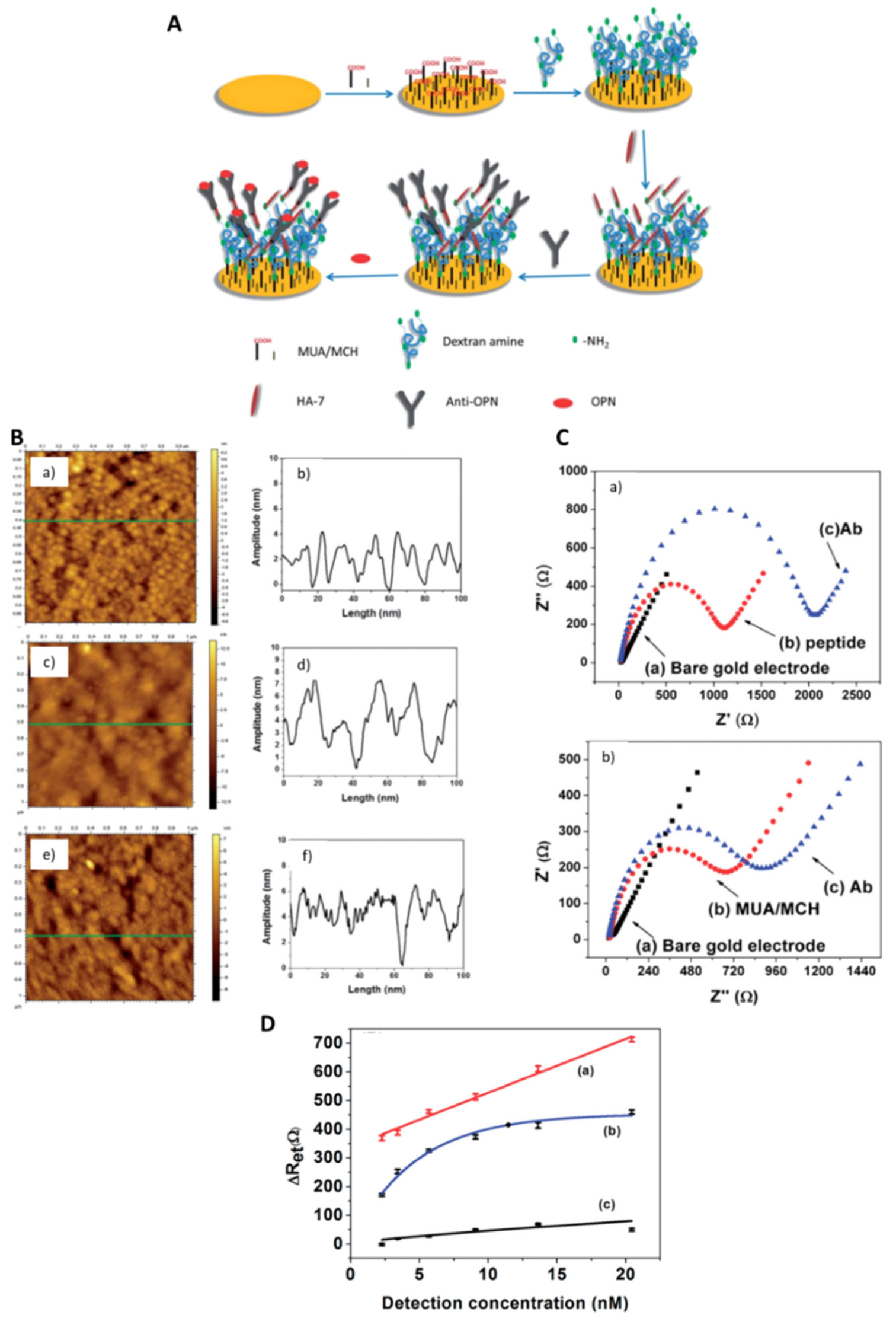

(caption on next page) 


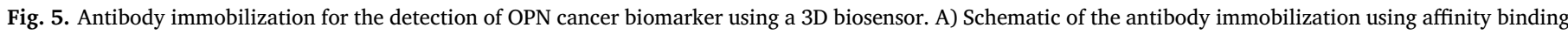

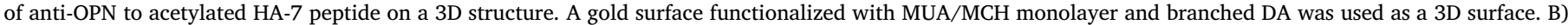

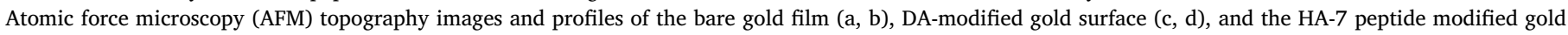

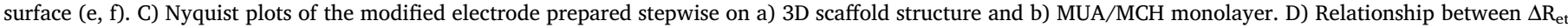

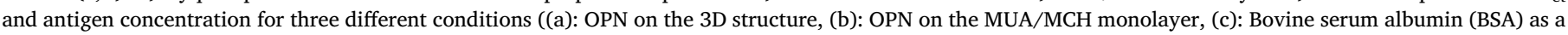
control on the 3D structure) (Chen et al., 2014). Figures adapted and reproduced with permission from: Royal Society of Chemistry.

orientation, impacted on the sensitivity and LoD of osteopontin (OPN) biomarkers detection. Consequently, the variation in the electron transfer resistance $\left(\Delta \mathrm{R}_{\mathrm{et}}\right)$ upon OPN detection was larger for the $3 \mathrm{D}$ scaffold at every concentration (Fig. 5D). Finally, a broader dynamic range was obtained for the 3D scaffold.

\subsection{Probe-free $3 D$ biosensors}

Although enzymes and antibodies provide high specificity and sensitivity to biosensors, 3D probe-free biosensors have also been reported, in particular for diabetes monitoring. These biosensors have the advantage of not relying on biomolecules that can lose their activity over time. This is possible due to material mechanisms, such as the swelling and stimuli response (Buenger et al., 2012; Deligkaris et al., 2010). On the other hand, these probe-free biosensors display reduced sensitivity and specificity compared to their immobilized counterparts. As an example, Lin et al. reported the development of a glucose-sensitive hydrogel that decreased the volume with the increment of glucose concentration due to the formation of reversible crosslinks. The differences in osmotic swelling pressure were detected by confining the hydrogel between a porous membrane and the diaphragm of a miniature pressure transducer (Lin et al., 2010). The main issue of enzymefree 3D biosensors is that PBA may bind to any molecule containing a cis-diol. Indeed, the binding affinity of PBA for fructose exceeds that for glucose. Nevertheless, strategies to overcome this issue have been reported (Tierney et al., 2009). MIPs are gaining an increased attention in the area of probe-free 3D biosensors since they are able to mimic natural recognition entities, like antibodies, proteins, and other biological receptors (see Fig. 1). MIPs are a versatile and promising technique that promotes selective cavities in a 3D polymeric network (Uzun and Turner, 2016; Vasapollo et al., 2011). As an example, Widayani et al. developed a 3D MIP biosensor based on methacrylic acid (MAA) using glucose as template. The response of the MIP-based electrode depended on the glucose concentration with a linear behavior from 0.02 to $5 \mathrm{mM}$. Unfortunately, this concentration range is not appropriate for clinical settings and further improvement may be required to improve its analytical performance (Widayani et al., 2017). Overall, even though probe-free biosensors are simpler compared to their probe-based counterparts, their performance is still very low, which threatens their use in the biomedical sector.

\section{Detection modes and transducers}

Table 2 shows some examples of biosensors for CVDs, cancer, and diabetes. As it can be observed, there is a diverse variety of detection modes, materials, and biomarkers that can be employed for disease detection, which determines the final performance of the biosensor. The selection of a specific biomarker depends on many factors. Importantly, among all available biomarkers, some are FDA-approved for clinical diagnosis, such as CA19.9 for pancreatic cancer, PSA for prostate cancer, or CEA for colorectal cancer, among others (Mordente et al., 2015). The interaction between the biomarker and the biorecognition element is transformed into a readable signal by the transducer. The transducer can be optical, piezoelectric, electrochemical, or electrochemiluminescence, among others. In general, biosensors can be classified according to their detection mode as electrochemical, piezoelectrical, optical and magnetic resonance relaxometry (Table 3) (Dambors et al., 2016; Fan et al., 2008; Frascella et al., 2016;
Grieshaber et al., 2008; Guenther, 2010). Detection modes are similar on both 2D and 3D biosensors. However, the final analytical performance is improved when using 3D platforms, since the signal is amplified by the 3D architecture. Further, many transducers can also display a 3D geometry, increasing the contact area between the biorecognition molecules and the signal detector.

\subsection{Electrochemical}

Most of the 3D biosensors used in the diagnosis and monitoring of CVDs, cancer, and diabetes use electrochemical transducers (Chen et al., 2014; Colombo et al., 2015; Devillers et al., 2017; Elshafey et al., 2013; Guenther, 2010; Ju et al., 2008; Kazemi et al., 2016; Kunduru et al., 2010; L. Li et al., 2015; Pedrosa et al., 2011; Rong et al., 2015; Sabury et al., 2015; Shan and Ma, 2017; Slaughter and Sunday, 2014; Zanghelini et al., 2017). This is mainly due to their simplicity, high sensitivity, and low production cost (Hammond et al., 2016). The first commercial electrochemical biosensor was reported in 1974. It was a Yellow Springs Instrument Company analyzer (Model 23A YSI) applied for the direct measurement of glucose, and was based on the amperometric detection of hydrogen peroxide. Since then, electrochemical biosensors have significantly evolved (Bhalla et al., 2016; Keyes et al., 1979). As an example, Ju et al. developed a 3D biosensor using an amperometric glucose transducer with a coiled Pt/Ir working electrode and an $\mathrm{Ag} / \mathrm{AgCl}$ reference electrode. The biosensor was exposed to increasing glucose concentrations $(5-15 \mathrm{mM})$ and the current responses were measured. The amperometric response was studied with and without a porous collagen scaffold covering the electrode, and no significant differences were observed in terms of current change. However, the biosensor integrating the collagen scaffold displayed a longer time to reach the equilibrium current (Ju et al., 2009, 2008).

An electrochemical technique commonly used in electrochemical biosensors is electrochemical impedance spectroscopy (EIS). EIS allows the measurement of the electrical properties of the material (conductivity/resistivity) detecting the electrical changes arising from the biorecognition at the electrode surface, such as protein immobilization or antibody-antigen reaction. Interestingly, EIS is a highly sensitive label-free methodology. This technique measures changes in impedance upon the application of a small sinusoidal potential (or current) of fixed frequency. This technique can be affected by several parameters, including surface roughness, surface fractal geometry, 3D geometric distributions, and heterogeneities at the molecule scale (Kafizas et al., 2017; Zanghelini et al., 2017). As an example, Zanghelini et al. used EIS to measure atypical glycemic profile of a cancerous mammalian cell. For that, a $\mathrm{TiO}_{2}$ butterfly-like membrane nanostructures $\left(\mathrm{TiO}_{2}-\mathrm{MN}\right)$ was used simultaneously as a working electrode and as biorecognition layer support.

\subsection{Optical}

Optical biosensors are based on the change in the optical properties of the material during the interaction of an optical signal with a biorecognition element (Dambors et al., 2016; Fan et al., 2008). 3D materials amplify changes in the optical properties when compared to planar surfaces, providing an increment in the analytical performance of biosensors. Optical biosensors can be classified as label or label-free. Most of the label biosensors use fluorescent-based dyes due to their intrinsic high sensitivity. Bornhoeft et al. developed a composite hydrogel-based 


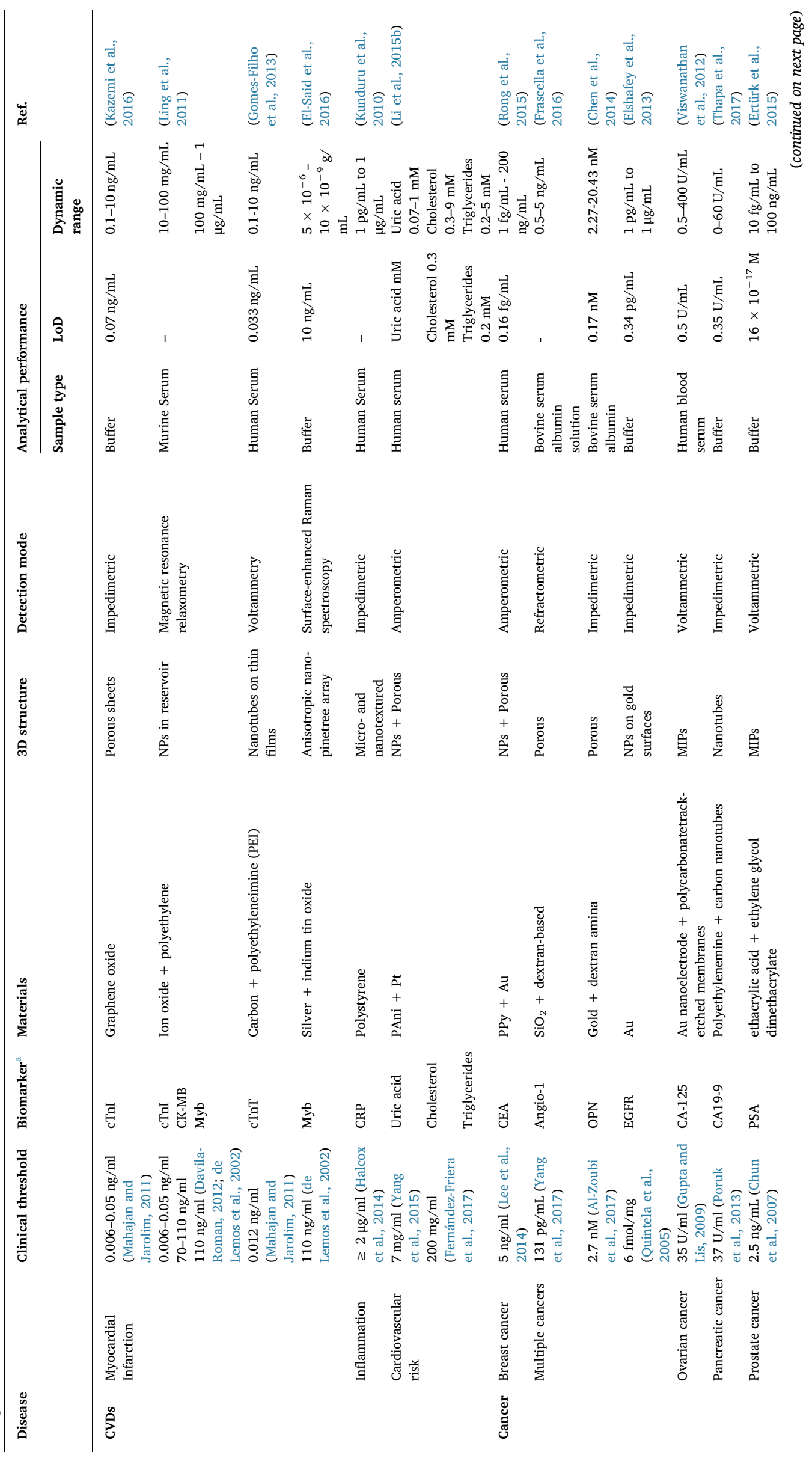




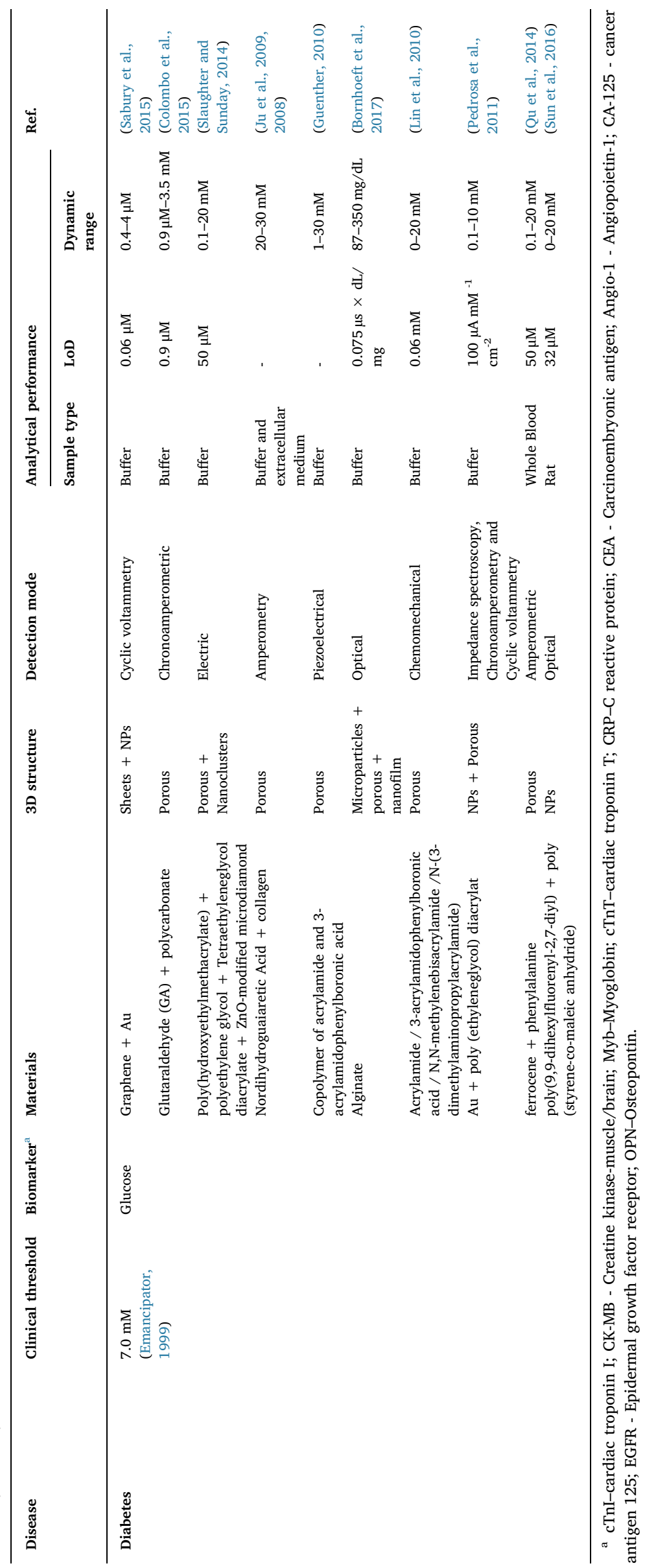


system, which was able to optically monitor glucose content. The composite system was formed by an alginate hydrogel embedded with alginate microparticles containing Pd-meso-tetra(4-carboxyphenyl) porphyrin (PdTCPP), $\mathrm{GO}_{\mathrm{x}}$ and catalase, and coated with PAH (poly (allylamine hydrochloride)) and poly(sodium-4-s tyrenesulfonate) (PSS) (Bornhoeft et al., 2017). PdTCPP is a dye responsive to glucose, through covalently immobilization of oxidoreductase enzymes and it is oxygen sensitive. Thus, PdTCPP is collisional quenched by molecular oxygen, being phosphorescence with lifetimes dependent and proportional to the local oxygen concentration. Therefore, the biosensor performance rely on the ability to control the diffusion of oxygen and glucose through the hydrogel composite. The optical measurements were performed in a custom-built instrument, with a LED excitement of $530 \mathrm{~nm}$ and a silicon photomultiplier detector. Emitted photons were collected in a ball lens, and the light was collimated and filtered before arriving to the detector (Bornhoeft et al., 2017).

\subsection{Piezoelectric}

Piezoelectricity is the ability of some dielectric materials to develop or change their electrical current when a mechanical strain is applied. The opposite is also true; if an electrical field is applied to piezoelectric materials, a mechanical strain is generated. According to these properties, a piezoelectric material can be used as an electromechanical transducer. Materials displaying a 3D architecture present a higher contact surface, which amplifies the piezoelectric signal. Interestingly, these materials do not require any external power supply, which makes them especially attractive for biosensor applications. Guenther et al. developed a 3D glucose biosensor using a piezoresistive hydrogel to monitor the analyte-induced volume expansion of a polymer layer, which worked as a chemo-mechanical transducer. A Wheatstone bridge was included on the hydrogel surface to transduce its deflection into an electrical output signal. This way, it was possible to continuously monitor the analyte-dependent swelling of a hydrogel, while the analyte concentration remained in ambient aqueous solution (Guenther, 2010). Thus, changes in environmental glucose concentration could be detected through the membrane pores and induce a change in the osmotic swelling pressure, allowing glucose monitoring (Lin et al., 2010).

\subsection{Magnetic resonance relaxometry}

This technique reports the measurement of relaxation variables in MRI. Despite being used as a non-invasive method of biosensing, resonance imaging is extremely expensive. This technique was used for the detection of $\beta$-human chorionic gonadotropin (a multiple cancer and cervical cancer biomarker) and cardiac biomarkers (cTnI, Myo and CK-MB-Creatine kinase-muscle/brain) detection, respectively, using a semi-permeable membrane and magnetic NPs to induce nanoparticle magnetic relaxation switches (MRSw). The semi-permeable membrane allowed the respective biomarkers to diffuse into the 3D biosensor, but not the diffusion of MRSw to tissue environment. MRSw in contact with biomarkers aggregated, decreasing the transversal relaxation time (Daniel et al., 2009; Ling et al., 2011). Note that this type of biosensors can only be used for a finite duration due to NPs saturation. Thus, it is extremely important to engineer NPs to match the sensitivity and lifetime of the chosen application.

\section{Integration of 3D biosensors into microfluidic devices: towards personalized medicine}

The integration of 3D biosensors into miniaturized microfluidic, or lab-on-a-chip, devices has the potential to revolutionize the field of point-of-care systems due to the combined advantages of their forming parts. This combination may provide clinicians with the measurement of clinical parameters from patients with ultrahigh precision in a fast, selective, and quantitative way. Importantly, the use of 
microfabrication techniques associated with lab-on-chip technology allows for the miniaturization, automatization, and parallelization of clinical assays and measurements, reducing the time required for the analysis and the required volume of sample and reagents. Overall, microfluidic systems are of high interest for pre-clinical experimentation and medical applications, which demand for novel devices with advanced properties capable to detect the presence of a specific compound in physiological fluids with high precision and to predict the response of drugs in patients. In this regard, organ-on-chip (OC) technology offers unprecedented possibilities for the development of a new generation of point-of-care systems with integrated biosensors, which allow treatment follow-up or better understanding of in vitro personalized disease models. OC are microfluidic devices, which contain human cells to mimic functional units of a tissue or an organ, recapitulating their biomolecular and hydrodynamic properties. OC emerged as a powerful tool to monitor in real-time the response of patients to specific therapies to diseases, such as CVDs, cancer, and diabetes, since they can easily integrate biosensor platforms (Caballero et al., 2017). These systems offer a precise control of the flow and microenvironment, which is essential for cell maintenance and growth, through a continuous supply of nutrients and removal of metabolic waste, better mimicking what happens in the human body (Halldorsson et al., 2015).

Even though the use of-2D-biosensors into microfluidic systems is well-established and a large body of literature is widely available, the integration of 3D biosensor technologies in OC devices is still far to be implanted. We examine herein the latest advances on the integration of 3D biosensors into OC systems for the monitoring of clinical-relevant biomarkers.

\subsection{Topographic-based biosensors}

As aforementioned, three-dimensional topographies include a diverse set of micro- and nano-sized structures, such as pillars, pores, cavities, grooves, spheres, and fibers (e.g., electrospun fibers, 3D graphene grown fibers, etc.) network, among others (see Fig. 1). These structures offer a large surface area for bioreceptor loading, which provides the biosensor platform with a higher sensitivity and selectivity compared to standard flat sensors. As an example, a microfluidic device was recently developed to create a microarray-based system to perform multiple on-chip immunoassays (Han and Koh, 2016). The microfluidic platform integrated a polysterene-based electrospun nanofiber matrix, acting as a 3D sensing scaffold, into which a large amount of fluorescently-labelled matrix metalloproteinase 9 (MMP-9) peptides were covalently immobilized. A solution containing MMP-9, an enzyme upregulated in several pathologies, such as in cancer (promoting angiogenesis, cancer cell invasion, and metastasis), was injected into the microfluidic device leading to the cleavage of a fragment of the labelled peptide, which diffused toward an observation chamber where the fluorescence signal was measured (Fig. 6A). A poly(ethylene)glycol hydrogel frame was used to provide the nanofibers matrix robustness and control the location of peptide immobilization, restricting the detection only into the microcavities containing the nanofibers. This system provided low LoD and offered an alternative to standard detection methods using antibodies. Even though the reported sensor selectivity and sensitivity might permit the measurement of MMP-9 within the physiological range, the current response time was still too long for realistic clinical applications. Future improvement to this work may include the culture of cancer cells/tissues in the 3D scaffold and the monitoring of MMP-9 directly from the produced extracellular matrix.

The secretion of factors out from cells is of high importance for a vast amount of physiopathological processes. Indeed, their detection is fundamental to assess the mechanistic determinants of diseases or disorders. However, this secretion critically depends on the observation or cell culture method. Microfluidic models mimic with higher fidelity the biological and rheological complexity of the native scenario and therefore, are an adequate method for the monitoring of cell secretion. Many sensing approaches have been used for such monitoring. Among them, microbeads display multiple advantages when integrated in 3D OC-based biosensors platforms, since they have the capacity to detect local concentrations of cell-secreted signals. Microbeads can either be immobilized onto the electrode surface, or be freely mobile within the microfluidic channel. In this regard, a fluorescent microbeads-based sensor was recently used combined with a microfluidic system for the detection of hepatocyte and transforming growth factors (HGF and TGF- $\beta 1$ ), respectively, secreted by a cell layer of primary hepatocytes. The cells and the sensing chamber were separated by an hydrogel that allowed secreted factors to diffuse unimpededly, while preventing cells from crossing over into sensing channels (Son et al., 2017). Indeed, these growth factors showed to have important roles in tumor development and progression. The secretion of growth factors was simultaneously monitored by measuring the emitted fluorescence at different time points using capture microbeads coated with anti-HGF and antiTGF- $\beta 1$. This allowed to obtain HGF and TGF- $\beta 1$ concentration in the order of pM (Fig. 6B).

\subsection{Entrapment-based biosensors}

The detection with high sensitivity and selectivity of molecules truly depends on the type of 3D transducer. Under certain circumstances, the detection probe needs to be completely surrounded by a matrix to provide the system with mechanical support, protecting the detection probes from degradation, and enhancing overall sensor sensitivity. In this regard, microfluidic 3D entrapment-based microfluidic biosensors emerged as an alternative to standard methodologies. As an example, Jang et al. developed a microfluidic device containing microchannels of $200 \mu \mathrm{m} \times 50 \mu \mathrm{m}$ to entrap GOx and peroxidase (POD) enzymes within a 3D PEG hydrogel microstructures, and silica-coated silver NPs (Ag@ $\mathrm{SiO}_{2}$ ) to enhance the fluorescence signal. As a proof of concept, a $10 \mathrm{mM}$ glucose solution was injected within the chip into different channels containing the PEG microstructures with and without Ag@ $\mathrm{SiO}_{2} \mathrm{NPs}$. The fluorescence signal was only observed on the latter, and importantly, only after $5 \mathrm{~min}$ after injection (Fig. 6C) (Jang et al., 2015). Glucose detection was also performed using a droplet-based microfluidic assay (Piao et al., 2015). In this work, water-in-air droplets containing a certain amount of glucose, HRP (Horseradish peroxidase), and Amplex Red probe were injected inside the chip, and the GOx enzyme was encapsulated into a hydrogel inside the microchannel. The droplets passed through the hydrogel where a catalytic reaction with the immobilized GOx converted glucose and oxygen to gluconolactone and $\mathrm{H}_{2} \mathrm{O}_{2}$, respectively, converting the Amplex Red substrates to the fluorescent products. The glucose level was quantitatively monitored by measuring in real-time the fluorescence signal of the droplets induced by the interaction of the released $\mathrm{H}_{2} \mathrm{O}_{2}$ with the HRP (Fig. 6D). The fluorescence signal was linear with the concentration of glucose, and concentrations up to $3 \mathrm{mM}$ could be detected, with a LoD of $10 \mu \mathrm{M}$. Interestingly, the reusability of the GOx/hydrogel was demonstrated, paving the way towards re-usable devices.

Even though entrapment-based microfluidic biosensors offer a large plethora of possibilities, they also present some drawbacks. Large biomolecules cannot diffuse within the hydrogels due to the relatively small mesh size network, limiting their interaction with the entrapped proteins (Lee et al., 2010). This limits the applicability of this method to small molecules, such as glucose and DNA, which are important for diabetes and cancer biomarker monitoring (Han et al., 2017). In this regard, a 3D hydrogel DNA microfluidic device was developed for the detection of single-nucleotide variants with high specificity and selectivity (Jung et al., 2015). The chip contained a sequence of photopolymerized 3D hydrogels into which the DNA detection probes were embedded. Electrophoresis was used to rapidly drive the hybridization of the target DNA towards the different hydrogel chambers containing 
A Before injection

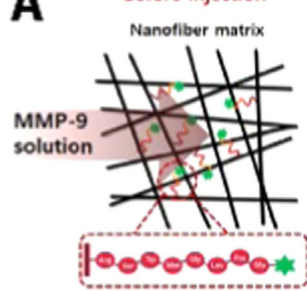

1000000
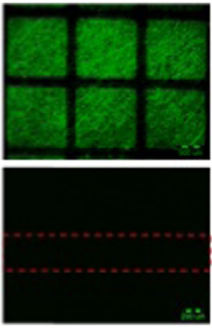

Nanofiber matrix

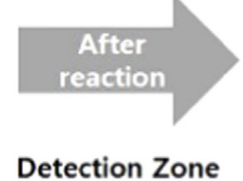

Detection Zone
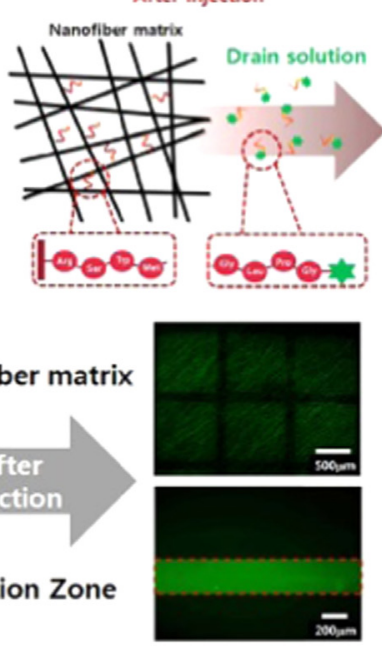

C
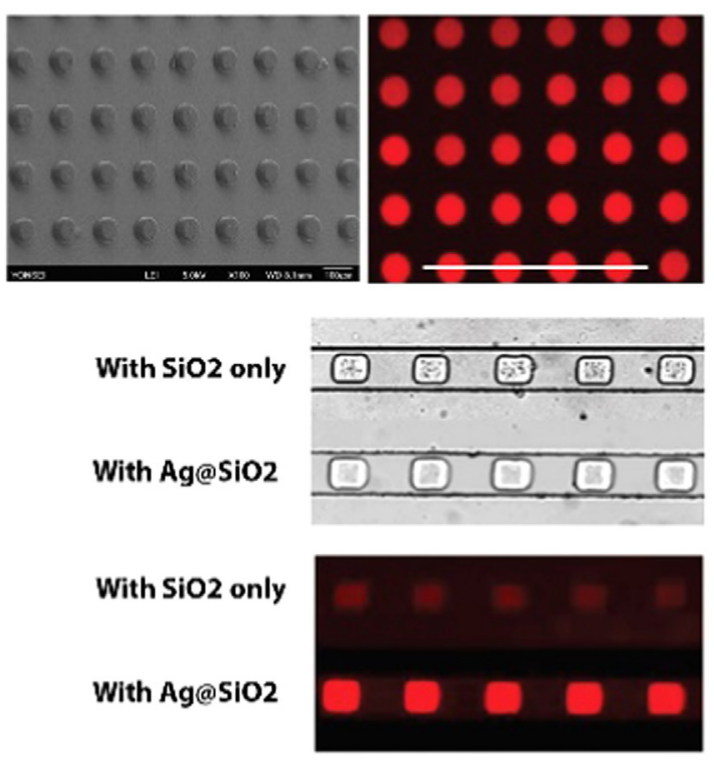

B

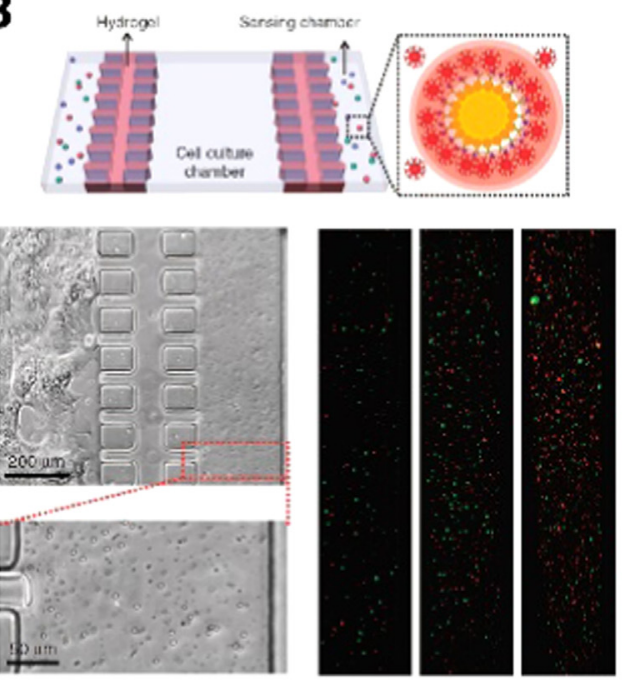

D

Glucose, Amplex Red, HRP

Resorufin (Ex 530 nm/Em $590 \mathrm{~nm}$ ) $\longrightarrow$

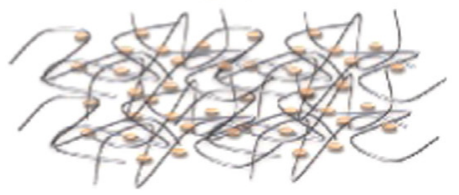

Hydrogel/GOx film

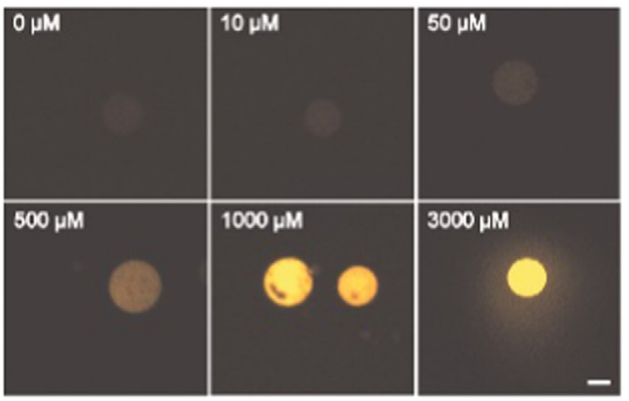

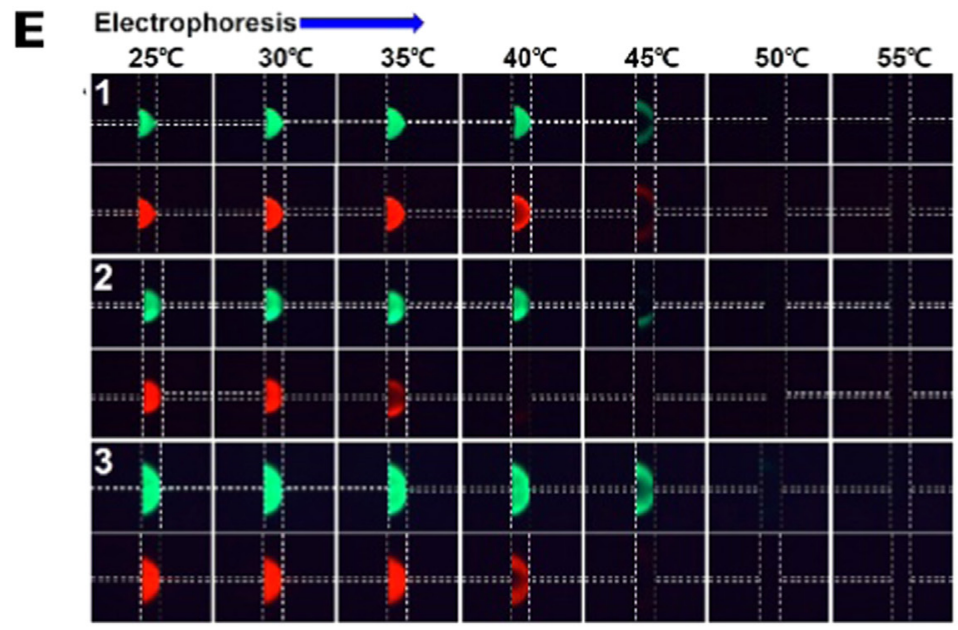




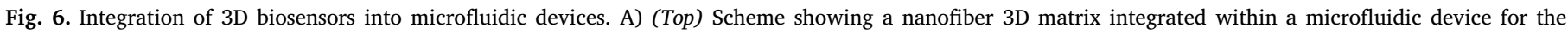

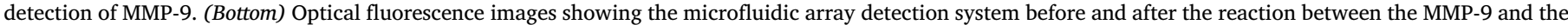

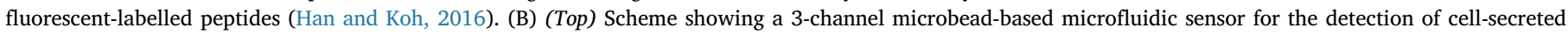

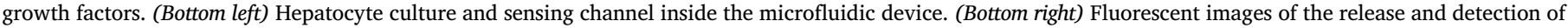

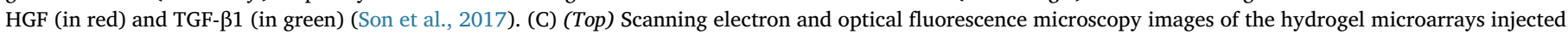

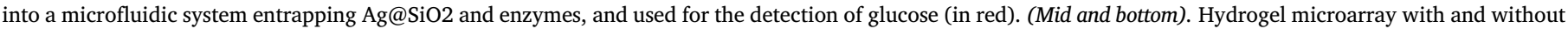

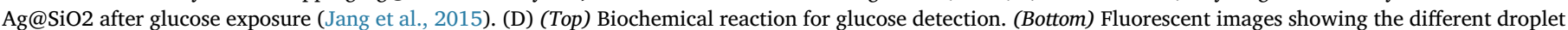

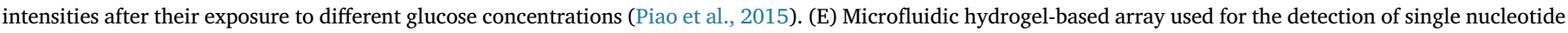

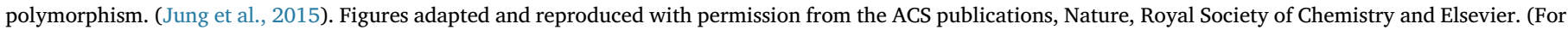
interpretation of the references to color in this figure legend, the reader is referred to the web version of this article.)

the capture DNA. Single-nucleotide mismatch detection was accomplished by performing temperature-gradient electrophoresis, using the difference in the melting of perfectly matching DNA $v$ s those displaying a mismatch (Fig. 6E). The device was successfully applied to target DNA with one base mismatch, displaying an impressive LoD of $1 \mathrm{pM}$ attributable to the high activity of the capture probes in the 3D capture hydrogels. On the other hand, the performance of the developed device, capable to only analyze single-stranded DNA targets, as well as the overall detection time of about $1 \mathrm{~h}$, limits the potential applicability of the developed device into the clinics. In a follow-up work, the microfluidic device was updated allowing the multiplexed detection of single nucleotide polymorphism of human clinical samples comprising double-stranded DNA. In this case, a LoD of $0.024 \mathrm{fM}$ was achieved using clinical samples (Jung et al., 2016).

\section{Implantable 3D biosensors}

The idea of having devices inside the human body that can diagnose a pathology early and monitor health condition can revolutionize medical care. However, implantable devices capable to detect, quantify, and transmit the information to the outside still remains a challenge. First, overcoming the foreign body response is considered one of the major limitations. Next, the response degree depends on the biosensor properties, such as its shape, size, roughness, porosity, surface chemistry, sterility, implantation duration, and/or degradation time (Onuki et al., 2008). Then, the maintenance of the activity of the immobilized probes and the matrix effect can greatly interfere with the analytical performance of the implanted biosensors. Lastly, the continuous monitoring of analytes, implies long-term stability of the biorecognition layer, a continuous dissociation of the analyte after the signaling event, and an effective storage/transmission of data values (Rogers and Boutelle, 2013; Sasso et al., 2013).

In an attempt to overcome all these challenges, Ju et al. developed a $3 \mathrm{D}$ porous collagen scaffold around an amperometric glucose biosensor. This was crosslinked with nordihydroguaiaretic acid (NDGA) or GA to improve its biocompatibility and biological stability. To evaluate their long-term stability, the glucose biosensor was implanted into rats and assessed weekly over a total of 4 weeks. The sensitivity of the biosensor dramatically decreased after implantation (when compared with the pre-implantation values), most probably a consequence of the tissue damage caused by the foreign body response. However, NDGA-crosslinked scaffolds presented a higher sensitivity than GA-crosslinked ones. The formers retained their physical structure with reduced inflammation when compared to GA-crosslinked scaffolds ( $\mathrm{Ju}$ et al., 2009).

A tight control is a fundamental requirement for the prevention, diagnostics and management of diseases like CVDs, cancer and diabetes (Ling et al., 2011). It is, therefore, crucial to use a biosensor with in vivo long-term stability capable of continuous monitoring while reducing the transducer degradation and problems associated to implantation (Sun et al., 2016). In order to overcome these limitations, Ling et al. developed a magnetic biosensor to continuously monitor changes in three different biomarkers (Fig. 7A). Superparamagnetic iron oxide NPs $(50 \mathrm{~nm})$ with amine-terminated dextran shells were coupled with monoclonal antibody against goat IgG. Then, the NPs were functionalized against specific targets through incubation with goatproduced polyclonal antibodies against cTnI, myoglobin and CK-MB and then encapsulated in high density polyethylene devices. The NPs aggregated around the analyte molecules and altered the transverse relaxation of adjacent water protons. The developed 3D biosensors were implanted in murine models and their performance analyzed. Biosensors presented LoDs in the range of $10-100 \mathrm{ng} / \mathrm{mL}$ for cTnI, and $100 \mathrm{ng} / \mathrm{mL}$ to $1 \mu \mathrm{g} / \mathrm{mL}$ to myoglobin and Ck-MB, which although it is not enough for clinical application, is significant considering measurements for in vivo performance. Nevertheless, it is important to note that biosensors saturated at $\approx 1.5 \mu \mathrm{gh} / \mathrm{mL}$, which is a huge constraint for the biosensor lifetime and cardiac monitoring duration (Ling et al., 2011). The same strategy was applied by Daniel et al. for the detection of hCG- $\beta$ cancer biomarker. Biosensors of high density polyethylene (HDPE) were produced and functionalized with MRSw coated with antihCG- $\beta 95$ and anti-hCG- $\beta 97$. Next, they were implanted in mice showing a detection range between 0.5 and $5 \mu \mathrm{g} / \mathrm{mL}$ hCG- $\beta$ and a LoD of $0.5 \mu \mathrm{g}$ / $\mathrm{mL}$. The evaluation of biosensor performance was limited by the lack of correspondence between the biomarker concentration in naturally occurring tumor and the xenograft tumor model used. In the latter, the biomarker concentration was significantly higher, which is not representative of the real physiological environment. Similarly, the longterm stability of the biosensor was not studied (Daniel et al., 2009).

With the aim of using less invasive techniques, Sun et al. developed a strategy for the real time and dynamic monitoring of small molecules in vivo by using a fluorescent semiconductor polymer dots (Pdot) oxygen transducer. GOx-functionalized Pdots were used for the continuous monitoring of glucose levels. Pdots transducer consisted in a semiconductor polymer (PDHF-Poly [9, 9-di-(2'-ethylhexyl) fluorenyl2,7-diyl]) doped with platinum octaethylporphyrin (PtOEP), an oxygen sensitive phosphorescent dye. The changes in oxygen concentration promoted by glucose levels were transduced into an optical signal (Sun et al., 2016). Next, Pdot-GOx NPs were implanted in a mice model for a period of 30 days through a subcutaneous injection (Fig. 7B). Due to their extraordinary brightness, the concentration of glucose in the microgram range was detectable transdermally. After the implantation time the biosensor luminescence still correlated with glucose levels and no damage was observed in the mice organs. However, a decrease in sensitivity was observed most likely due to a decrease in enzyme's catalytic activity in Pdots surface. Despite the promising results the long-term monitoring was studied only for 30 days, which is still a short period of time (Sun et al., 2016).

Actual examples of 3D biosensors employed for clinical applications are scarce. Many reasons may be behind this low adoption rates, such as their higher complexity compared to standard-planar-technologies, the lack of standardization or certification (e.g., FDA-approved), and in particular for implantable devices. As aforementioned, it is not yet not well understood how foreign body response (e.g., inflammation) on implanted devices may hamper the clinical biomarkers detection. In addition, it needs to be investigated whether the formation of a fibrous capsule surrounding the biosensor may cause a significant loss of its performance over time. Next, the swelling of 3D biomaterials (e.g. hydrogels), their permeability to different molecules, their interaction with different media, and the optimal immobilization of biomolecules may need to be optimized to ensure an optimal biosensor performance. 
A

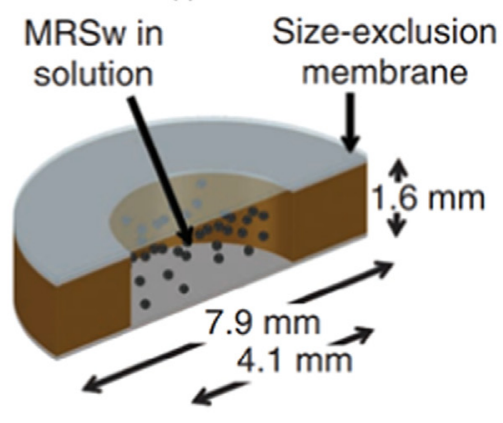

B

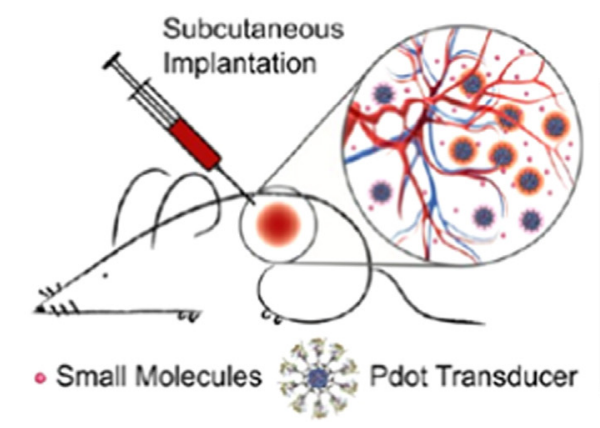

(ii)

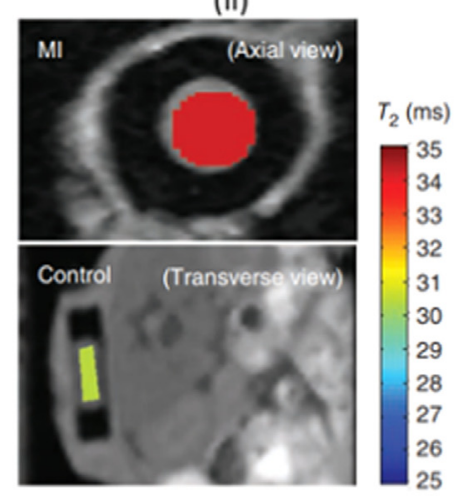

(ii)

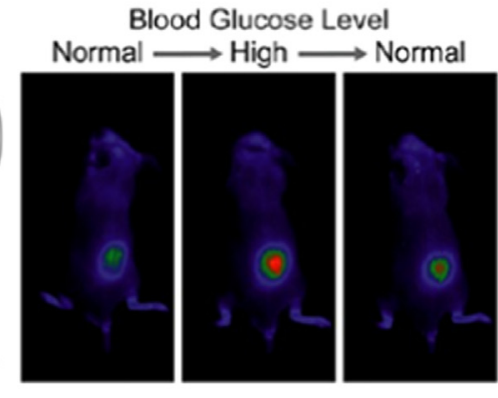

Fig. 7. Implantable 3D biosensors. A) Magnetic resonance relaxometry 3D implantable biosensor. (i) The sensor consists on a reservoir containing MRSw particles enclosed by a size-exclusion membrane. (ii) Feasibility of MRI-based in situ measurements after $24 \mathrm{~h}$ implantation with acute myocardial infarction (MI) and without (control) (Ling et al., 2011). B) Pdot-GOx biosensor function mode. (i) Schematic of insertion of a Pdot-GOx biosensor and Pdot transducer function mode. (ii) Fluorescence images of different glucose concentrations of the implanted biosensor by luminescence intensity (Sun et al., 2016). Figures adapted and reproduced with permission of Nature and ACS publications.
To address all these challenges several clinical trials are currently under investigation aiming to introduce biosensors in the clinics in the near future (see also Sections 7 and 8). The successful widespread application of 3D biosensors in current clinical applications will definitely require contributions from different fields, such as microelectronics and nanotechnology. This will help to standardize all the manufacturing processes and to provide the needed stability and reliability requested by the clinicians and pharmaceutical companies (Carpenter et al., 2018; Hasan et al., 2014; Zhu et al., 2015). Finally, the use of new approaches in signal transduction, such as wireless, polymer-dots or magnetic particles may boost their adoption by the healthcare market.

\section{Commercialization and regulatory issues: from lab to the market}

The development of 3D biosensors, and their integration into implantable medical devices and OC-based disease models, have the potential to offer great market opportunities to pharmaceutical and biotechnological companies. Similarly, the reduction of the costs associated to drug discovery and screening, and the increase in the reliability of drug testing, makes 3D biosensors also appealing for clinical applications. The national healthcare systems may also benefit from their development by reducing the high cost associated to public healthcare. However, for this to become a reality, many constrains and challenges need to be addressed. These challenges are similar to those encountered by planar sensing platforms, which are mainly technical and have been broadly discussed above. However, the integration of 3D biosensors into implantable medical devices is additionally associated with a major constrain: the complex regulatory issues for clinical products. For clinical application, medical devices must go through a long and careful regulation process before commercialization to ensure the device safety under the strict supervision of the regulatory agencies (FDA-Food and Drug Administration in US, and CE-European Conformity in EU) (Fiedler and Ferguson, 2017; Mazzocchi, 2016; Meng, 2014; Pina et al., 2018). Usually, it begins by the development of the product in the laboratory under GMP (good manufacturing practices) conditions, followed by animal testing in a highly controlled environment, and finally, clinical trials. In this regard, a thorough survey using several international databases (Clinicaltrials.gov, the International Clinical Trials Registry Platform-WHO, the European Organization for Research and Treatment of Cancer-EORTC, the EU Clinical Trials Register, the European Cancer Patient Coalition-ECPC, and the Australian New Zealand Clinical Trials Registry-ANZCTR) reported several clinical trials around the world of biosensors for cancer, CVDs, and diabetes applications. However, in most of the cases, very few information about the biosensor characteristics was reported (see Table 4).

The absence of product development guidelines also contributes to the low level of development of medical devices integrating 3D biosensors. Indeed, 3D biosensors research still need to work towards improving the long-term performance in complex biological environments. However, there is a global effort to increase the investment levels in translational research, in particular for healthcare applications. This effort is beginning to be recognized by the number of patents $(\sim 50)$ regarding 3D biosensors in the last few years (WIPO database) with potential industrial and clinical applications (Table 5). Similarly, several high-tech, biotechnological and pharmaceutical companies are investing a large amount of resources in developing diagnostic and biosensing devices, such as Medtronic ${ }^{\mathrm{TM}}$, Phillips ${ }^{\mathrm{TM}}$, Abbott $^{\mathrm{TM}}$, Dexcom ${ }^{\mathrm{TM}}$, Endotronix $^{\mathrm{TM}}$, CellNovo ${ }^{\mathrm{TM}}$, iRhythm Technologies ${ }^{\mathrm{TM}}$, LivaNova PLC, Roche $^{\mathrm{TM}}$, or Profusa ${ }^{\mathrm{TM}}$, among many others. The latter is developing a porous hydrogel linked to a light fluorescent-emitting molecule that produces a signal in the presence of glucose or any other biomarker of interest, which is monitored by a separated optical reader. Interestingly, this device can be adhered to the skin or held by hand ("Profusa, Inc.," n.d.). Other companies are also commercializing their own products (e.g., implantable cardiac defibrillators (Medtronic ${ }^{\mathrm{TM}}$ ), Cardiomems $^{\mathrm{TM}}$ heart failure monitoring system (FDA-approved; Abbott), the Cordella $^{\mathrm{TM}}$ heart failure sensor (Endotronix ${ }^{\mathrm{TM}}$ ), or the Enlite ${ }^{\mathrm{TM}}$ glucose sensor (Medtronic ${ }^{\mathrm{TM}}$ )), or evaluating their performance in the clinical trials (see Table 4). In this regard, most of the reported clinical trials are related to heart failure monitoring and stimulation and for the 
Table 4

Most relevant clinical trials on biosensors for CVDs, cancers, and diabetes.

\begin{tabular}{|c|c|c|c|c|}
\hline Trial ID (Year) & Application & Status & Title & Comment \\
\hline $\begin{array}{l}\text { NCT02545166 } \\
(2016-2019)\end{array}$ & CVD & Active & $\begin{array}{l}\text { Validation of a Purine Biosensor in Detecting Acute Cerebral } \\
\text { Ischaemia: Carotid Endarterectomy Model in SMARTChip (CEMS) }\end{array}$ & In vitro; detection of blood purine levels. \\
\hline $\begin{array}{l}\text { NCT02308605 } \\
(2014-2016)\end{array}$ & CVD & Completed & $\begin{array}{l}\text { SMARTCap Stroke Study: A Field Deployable Blood Test for Stroke } \\
\text { (SMARTCAP) }\end{array}$ & $\begin{array}{l}\text { In vitro; detection of blood purine levels (early } \\
\text { biomarker of brain ischaemia). }\end{array}$ \\
\hline $\begin{array}{l}\text { NCT03247829 } \\
(2017-2019)\end{array}$ & CVD & Active & $\begin{array}{l}\text { Investigation to Optimize Hemodynamic Management of Left } \\
\text { Ventricular Assist Devices Using the CardioMEMS }\end{array}$ & $\begin{array}{l}\text { Implanted; direct pulmonary artery pressure } \\
\text { monitoring; FDA-approved. }\end{array}$ \\
\hline $\begin{array}{l}\text { NCT03375710 } \\
(2017-2020)\end{array}$ & CVD & Active & SIRONA Trial Heart Failure NYHA Class III & $\begin{array}{l}\text { Implanted; pulmonary artery pressure sensor; } \\
\text { first-in-human implantation. }\end{array}$ \\
\hline $\begin{array}{l}\text { NCT02957370 } \\
(2015-2020)\end{array}$ & Cancer & Active & Molecular Biosensors for Detection of Bladder Cancer & In vitro; detection of urinary anomalies. \\
\hline $\begin{array}{l}\text { NCT02195076 } \\
(2014-2017)\end{array}$ & Cancer & Completed & Non Invasive Detection of Lung and Breast Cancer by Odor Signature & Ex vivo; detection of volatile compounds. \\
\hline $\begin{array}{l}\text { NCT03173729 } \\
(2017-2022)\end{array}$ & Cancer & Active & $\begin{array}{l}\text { Point of Care, Real-time Urine Metabolomics Test to Diagnose } \\
\text { Colorectal Cancers and Polyps in Low and Middle Income Countries }\end{array}$ & In vitro; detection of urine biomarkers. \\
\hline $\begin{array}{l}\text { NCT03445065 } \\
(2018-2019)\end{array}$ & Diabetes & Active & $\begin{array}{l}\text { Benefits of a Long Term Implantable Continuous Glucose Monitoring } \\
\text { System for Adults With Diabetes - France Randomized Clinical Trial }\end{array}$ & $\begin{array}{l}\text { Implanted (subcutaneous); continuous } \\
\text { monitoring of glucose levels. }\end{array}$ \\
\hline $\begin{array}{l}\text { NCT01991470 } \\
(2015-2016)\end{array}$ & Diabetes & Completed & $\begin{array}{l}\text { A Performance Evaluation of the Enlite }{ }^{\mathrm{TM}} \text { and Enlite } 3 \text { Glucose Sensor } \\
\text { to Support Use in Children }\end{array}$ & \\
\hline $\begin{array}{l}\text { NCT01065948 } \\
(2009-2012)\end{array}$ & Diabetes & Completed & $\begin{array}{l}\text { A Feasibility Study to Assess Critical Aspects of Fluorescence Affinity } \\
\text { Sensor (FAS) Performance and Safety Over Several Hours }\end{array}$ & \\
\hline $\begin{array}{l}\text { ACTRN12617000919314 } \\
(2017)\end{array}$ & Diabetes & To be opened & $\begin{array}{l}\text { Investigation of a long-term continuous glucose monitor in type } 1 \\
\text { diabetes }\end{array}$ & \\
\hline
\end{tabular}

Table 5

Most significant patents in the 3D biosensors field.

\begin{tabular}{|c|c|}
\hline Patent Number (Year) & Title \\
\hline $\begin{array}{l}\text { US20180045681 } \\
(15.02 .2018)\end{array}$ & $\begin{array}{l}\text { System and method of measuring cell viability and } \\
\text { growth. }\end{array}$ \\
\hline $\begin{array}{l}\text { KR1020160124013 } \\
(26.10 .2016)\end{array}$ & Biosensor for monitoring 3D cell culture in real time. \\
\hline $\begin{array}{l}\text { US20140011697 } \\
(09.01 .2014)\end{array}$ & $\begin{array}{l}\text { 3D RF MEMS biosensor for multiplexed label free } \\
\text { detection. }\end{array}$ \\
\hline $\begin{array}{l}\text { US20130206595 } \\
(15.08 .2013)\end{array}$ & $\begin{array}{l}\text { Biosensor with three-dimensional structure and } \\
\text { manufacturing method thereof. }\end{array}$ \\
\hline $\begin{array}{l}\text { US20100066346 } \\
(18.03 .2010)\end{array}$ & $\begin{array}{l}\text { Fabrication of microstructures integrated with } \\
\text { nanopillars along with their applications as electrodes } \\
\text { in sensors. }\end{array}$ \\
\hline $\begin{array}{l}\text { EP2088430 } \\
(12.08 .2009)\end{array}$ & $\begin{array}{l}\text { Biosensor having 3D metallic nanowire electrodes } \\
\text { forming nanochannel, manufacturing method thereof, } \\
\text { and bio disk system having same. }\end{array}$ \\
\hline
\end{tabular}

continuous monitoring of the glucose levels. In contrast, few cancerrelated devices, in particular, with implantable characteristics have been described.

\section{Unmet needs and future perspectives}

The current level of development of 3D biosensors makes their translation to the clinics in the near future unrealistic despite some successful exceptions (see Tables 4 and 5). In particular, it is not yet clear whether 3D biosensor can actually solve the limitations of current biosensing technologies in the clinical settings. One of the main reasons is that typically, most of the tests for in vitro biomarker detection performed in the laboratory use simplified matrices and not physiological samples, which reduce the sensitivity and selectivity of biosensors. In fact, even though 3D biosensors display suitable analytical performance in the laboratory settings, they are still far from being applied in the clinics in a regular basis, in particular, as implantable devices. Several serious limitations 3D biosensors must address before being adopted, include their multiplex capability, reproducibility, assay duration, stability, or the exploration of novel biomarkers, among others. Multiplex capability can easily be achieved using microfluidic in vitro system, where multiple microchannels and 3D chambers could be integrated together with the sensing entities. This allows the simultaneous detection of several analytes (Misun et al., 2016). Similarly, static 3D biosensor may also display multiplex capabilities. To this aim, an array of 3D porous structures or topographic micro/nano-structures may be employed, with several regions containing the different biorecognition elements. Indeed, these approaches were recently employed for the detection of cancer and cardiovascular biomarkers, resulting in a performance similar to the one obtained with standard and time-consuming laboratory techniques (Nikitin et al., 2018), and importantly, reducing the time of the assay. However, their in vivo multiplex performance still remains a challenge. The use of transdermal 3D biosensor integrating multiple 3D micro/nano-needles (each of them detecting the different analyte) may provide a plausible solution to the multiplex capabilities of the implantable devices.

3D biosensors may also improve the reproducibility of the assays, a pitfall in the biosensors field, by increasing the stability of the biorecognition layer. In planar biosensor platform, this layer is more exposed to the harsh environment in contrast to 3D biosensors, where the layer is more protected, in particular in porous 3D structures or hydrogels. In addition, 2D biosensors are more prompt to saturation and to the adhesion of non-specific proteins (corona effect). Due to the enhance surface-to-volume ratio of 3D biosensors, which also increases the number of capture probes, they are expected to display less variability and fluctuations in the measurements, thus contributing to an enhanced reproducibility of the data. In this regard, real-time continuous measurement is also a major challenge of current biosensing techniques; the response cycles are still too long and the signal stability is still not robust enough. As aforementioned, the superior architecture of 3D biosensors may increase sensor sensitivity and selectivity, thus allowing real-time measurements.

Next, 3D biosensors may also allow exploring, revisiting, and using new-unexpected-biomarkers considered as non-adequate for planar biosensing technologies. The enhanced sensitivity of 3D biosensors may allow re-using biorecognition elements discarded, thus offering a larger catalogue of capture probes. In particular, this may be fundamental for diabetes, since most of the studies are based on the periodic detection of glucose levels in blood. The use of alternative biomarkers compatible with other body fluids (e.g., saliva) may open up the new avenues, which may avoid blood extraction using (semi)-invasive methods (Ladgotra et al., 2016; Srinivasan et al., 2015).

In general, cancer and diabetes are more chronic diseases than CVDs. The formers require high sensitivity and specificity for their early detection and prognosis, whereas the latter require a fast assay time. In this regard, the needs for detection of these diseases are not exactly the 
same, thus 3D biosensor must display a large plethora of possibilities in terms of configuration, materials, and analytical performance. This allows their use in a wide variety of physiopathological processes. For cancer applications, the enhanced sensitivity given by the 3D architecture of the electrode/biorecognition layer provides the needed sensitivity and specificity. In addition, the multiplex capability mentioned above allows a multi-parametric detection of analytes associated with the disease at early stages. This also holds true for diabetes, where the enhanced sensitivity may prevent the measurement of glucose levels in blood by measuring the presence of biomarkers, for example, in saliva. Finally, for CVDs, the use of implantable devices allows for a continuous monitoring of heart activity capable to detect and prevent heart failure.

Finally, it is yet not well understood the reaction kinetics occurring on the electrode in 3D biosensors, as well as other physicochemical phenomena (electron transfer kinetics, double layer capacitance, Warburg-diffusion-impedance, and others), which may affect the signal recorded on the electrode. This is in particular fundamental in highly porous structures in order to understand the origin of the detected signal. Some works have reported on the electrochemical behavior of biosensors depending on the structure of 3D pore electrode, showing the critical relation between diffusion and biosensor performance (Ye et al., 2015). Similarly, it was shown that the interplay between the pore size, and the coating and electrode thickness plays a fundamental role for improved sensor response. The impact of enzyme distribution in 3D porous electrodes has been recently addressed (Mazurenko et al., 2017). Typically, enzyme distribution has been considered homogeneous. In this work, it was shown that only $10-15 \%$ of the total quantity of adsorbed enzymes contributed to the catalysis. Further, simulation studies have been performed for a rational design of 3D porous electrodes with enhanced selectivity and sensitivity (Halhouli et al., 2016). In this case, not only the size but also the number of pores influence the diffusion within the electrode due to an enhanced diffusion resistance.

\section{Conclusions}

Cardiovascular diseases, cancer and diabetes are worldwide high rate mortality diseases. Their early prognosis urge new medical technologies able to continuously screen and monitor specific biomarkers and to overcome the limitations associated to standard 2D-based biosensing technologies. 3D biosensors offer a large plethora of advantages when compared to flat biosensor devices, in particular in terms of the improved sensitivity and stability. The 3D architecture of the electrodes maximizes the amount of immobilized probes and the electron transfer, which contribute for the improved performance. They also offer enhanced implantable characteristics, fundamental for the continuous and real time monitoring of the disease. They may also be integrated into physiologically-relevant OC platforms to evaluate and predict the efficiency of personalized treatments with high sensitivity and reliability prior moving to the patient. These innovative technologies demand high performance, flexible, and biocompatible 3D biosensor platforms. In this regard, a new generation of 3D biosensors have emerged. However, moving to the third dimension in biosensors is associated with the appearance of critical physicochemical phenomena, which need to be fully considered if intended to improve biosensors performance. In this way, 3D biosensors will be capable to revolutionize disease detection, monitoring and treatment follow-up of high mortality diseases. Nevertheless, further integration of biosensing technology with tissue engineering approaches needs to be accomplished, translating the results obtained in the lab into commercially viable products. This is a long and judicious process, in which strict and specific requirements need to be fulfilled, under the severe guidance of international regulatory entities, before its implementation into the medical market.

\section{Acknowledgements}

V.M.C., S.C.K, and D.C. acknowledge the financial support from the European Union Framework Programme for Research and Innovation Horizon 2020 on Forefront Research in 3D Disease Cancer Models as in vitro Screening Technologies (FoReCaST) under Grant agreement no. 668983. V.M.C also thanks the Portuguese Foundation for Science and Technology (FCT) for his distinction attributed under the FCT Investigator program (IF/01214/2014). D.C. and S.C.K also acknowledge the support from the FCT under the scope of the project Modelling Cancer Metastasis into the Human Microcirculation System using a Multi-organ-on-a-Chip Approach (2MATCH) (PTDC/BTM-ORG/28070/ 2017) funded by the Programa Operacional Regional do Norte supported by Fundo Europeu de Desenvolvimento Regional (FEDER). A.I.B. acknowledges the financial support of project FROnTHERA (NORTE01-0145-FEDER-000023).

\section{Conflicts of interest}

None.

\section{References}

Ahmed, E.M., 2015. Hydrogel: preparation, characterization, and applications: a review. J. Adv. Res. 6, 105-121.

Ahmed, M.U., Saaem, I., Wu, P.C., Brown, A.S., 2014. Personalized diagnostics and biosensors: a review of the biology and technology needed for personalized medicine. Crit. Rev. Biotechnol. 34, 180-196. https://doi.org/10.3109/07388551.2013. 778228.

Akhtar, M.F., Hanif, M., Ranjha, N.M., 2016. Methods of synthesis of hydrogels ... a review. Saudi Pharm. J. 24, 554-559.

Al-Zoubi, S., Wassouf, A., Zetoune, A.B., 2017. Measuring levels of osteopontin as potential biomarker for hepatocellular carcinoma in Syrian patients. Gastroenterol. Hepatol. Bed Bench 10, 97-101.

Arora, S., Kaur, H., Kumar, R., Kaur, R., Rana, D., Rayat, C.S., Kaur, I., Arora, S.K., Bubber, P., Bharadwaj, L.M., 2014. In vitro cytotoxicity of multiwalled and singlewalled carbon nanotubes on human cell lines. Fuller. Nanotub. Carbon Nanostruct. 23, 377-382. https://doi.org/10.1080/1536383X.2013.812638.

Barbosa, A.I., 2015. The Development and Optimisation of a Novel Microfluidic Immunoassay Platform for Point of Care Diagnostics. Loughborough University.

Barbosa, A.I., Reis, N.M., 2017. A critical insight into the development pipeline of microfluidic immunoassay devices for the sensitive quantitation of protein biomarkers at the point of care. Analyst 142, 858-882. https://doi.org/10.1039/c6an02445a.

Berti, F.V., Srisuk, P., da Silva, L.P., Marques, A.P., Reis, R.L., Correlo, V.M., 2017. Synthesis and characterization of electroactive gellan gum spongy-like hydrogels for skeletal muscle tissue engineering applications. Tissue Eng. Part A 23, 968-979. https://doi.org/10.1089/ten.TEA.2016.0430.

Bertok, T., Sediva, A., Vikartovska, A., Tkac, J., 2014. Comparison of the 2D and 3D nanostructured lectin-based biosensors for in situ detection of sialic acid on glycoproteins. Int. J. Electrochem. Sci. 9, 890-900.

Bhalla, N., Jolly, P., Formisano, N., Estrela, P., 2016. Introduction to biosensors. Essays Biochem. 60, 1-8. https://doi.org/10.1016/B978-0-12-803100-1.00001-3.

Bornhoeft, L., Biswas, A., McShane, M., 2017. Composite hydrogels with engineered microdomains for optical glucose sensing at low oxygen conditions. Biosensors 7, 8 . https://doi.org/10.3390/bios7010008.

Borole, D.D., Kapadi, U.R., Mahulikar, P.P., Hundiwale, D.G., 2012. Conducting polymers: an emerging field of biosensors. Des. Monomers Polym. 9, 1-11.

Buenger, D., Topuz, F., Groll, J., 2012. Hydrogels in sensing applications. Prog. Polym. Sci. 37, 1678-1719. https://doi.org/10.1016/j.progpolymsci.2012.09.001.

Caballero, D., Kaushik, S., Correlo, V.M., Oliveira, J.M., Reis, R.L., Kundu, S.C., 2017. Organ-on-chip models of cancer metastasis for future personalized medicine: from chip to the patient. Biomaterials 149, 98-115. https://doi.org/10.1016/J. BIOMATERIALS.2017.10.005.

Carpenter, A.C., Paulsen, I.T., Wiliams, T.C., 2018. Blueprints for biosensors: Design, limitations, and applications. Genes 9, 375.

Cassell, A.M., Ye, Q., Cruden, B.A., Li, J., Sarrazin, P.C., Ng, H.T., Han, J., Meyyappan, M., 2004. Combinatorial chips for optimizing the growth and integration of carbon nanofibre based devices. Nanotechnology 15, 9-15. https://doi.org/10.1088/09574484/15/1/002.

Chambers, J.P., Arulanandam, B.P., Matta, L.L., Weis, A., Valdes, J.J., 2008. Biosensor recognition elements. Curr. Issues Mol. Biol. 10, 1-12.

Chen, H., Mei, Q., Jia, S., Koh, K., Wang, K., Liu, X., 2014. High specific detection of osteopontin using a three-dimensional copolymer layer support based on electrochemical impedance spectroscopy. Analyst 139, 4476-4481. https://doi.org/10. 1039/c4an00576g.

Chun, F.K.-H., Hutterer, G.C., Perrotte, P., Gallina, A., Valiquette, L., Benard, F., McCormack, M., Briganti, A., Ionescu, C., Jeldres, C., Guay, J.-P., Saad, F., Karakiewicz, P.I., 2007. Distribution of prostate specific antigen (PSA) and percentage free PSA in a contemporary screening cohort with no evidence of prostate 
cancer. BJU Int. 100, 37-41. https://doi.org/10.1111/j.1464-410X.2007.06923.x.

Colombo, L., Baruzzi, A.M., Garay, F.S., 2015. Analysis and optimization of a hydroge matrix for the development of a sandwich-type glucose biosensor. Sens. Actuators B Chem. 211, 125-130. https://doi.org/10.1016/j.snb.2015.01.063.

da Silva, L.P., Cerqueira, M.T., Sousa, R.A., Reis, R.L., Correlo, V.M., Marques, A.P., 2014. Engineering cell-adhesive gellan gum spongy-like hydrogels for regenerative medicine purposes. Acta Biomater. 10, 4787-4797. https://doi.org/10.1016/j.actbio. 2014.07.009.

Dambors, P., Svitel, J., Katrlík, J., 2016. Optical biosensors. Essays Biochem. 60, 91-100. https://doi.org/10.1042/EBC20150010.

Daniel, K.D., Kim, G.Y., Vassiliou, C.C., Galindo, M., Guimaraes, A.R., Weissleder, R., Charest, A., Langer, R., Cima, M.J., 2009. Implantable diagnostic device for cancer monitoring. Biosens. Bioelectron. 24, 3252-3257. https://doi.org/10.1016/j.bios. 2009.04.010.

Darne, C., Terzetti, F., Coulais, C., Fontana, C., Binet, S., Gaté, L., Guichard, Y., Guichard, Y., 2014. Cytotoxicity and genotoxicity of panel of single- and multiwalled carbon nanotubes: in vitro effects on normal Syrian hamster embryo and immortalized v79 hamster lung cells. J. Toxicol. 2014, 872195. https://doi.org/10.1155/2014/ 872195.

Davila-Roman, V.G., 2012. Cardiac biomarkers: expert advice for clinicians. Circulation 126, e264-e265. https://doi.org/10.1161/CIRCULATIONAHA.112.102855.

Deligkaris, K., Tadele, T.S., Olthuis, W., van den Berg, A., 2010. Hydrogel-based devices for biomedical applications. Sens. Actuators B Chem. 147, 765-774. https://doi.org/ 10.1016/j.snb.2010.03.083.

Derkus, B., Emregul, K.C., Emregul, E., 2015. Evaluation of protein immobilization capacity on various carbon nanotube embedded hydrogel biomaterials. Mater. Sci. Eng. C 56, 132-140. https://doi.org/10.1016/j.msec.2015.06.022.

Devillers, M., Ahmad, L., Korri-Youssoufi, H., Salmon, L., 2017. Carbohydrate-based electrochemical biosensor for detection of a cancer biomarker in human plasma. Biosens. Bioelectron. 96, 178-185. https://doi.org/10.1016/j.bios.2017.04.031.

Edmondson, R., Broglie, J.J., Adcock, A.F., Yang, L., 2014. Three-dimensional cell culture systems and their applications in drug discovery and cell-based biosensors. Assay Drug Dev. Technol. 12, 207-218. https://doi.org/10.1089/adt.2014.573.

El-Said, W.A., Fouad, D.M., El-Safty, S.A., 2016. Ultrasensitive label-free detection of cardiac biomarker myoglobin based on surface-enhanced Raman spectroscopy. Sens. Actuators B Chem. 228, 401-409. https://doi.org/10.1016/j.snb.2016.01.041.

Elshafey, R., Tavares, A.C., Siaj, M., Zourob, M., 2013. Electrochemical impedance immunosensor based on gold nanoparticles-protein $\mathrm{G}$ for the detection of cancer marker epidermal growth factor receptor in human plasma and brain tissue. Biosens. Bioelectron. 50, 143-149. https://doi.org/10.1016/j.bios.2013.05.063.

Emancipator, K., 1999. Laboratory diagnosis and monitoring of diabetes mellitus. Am. J. Clin. Pathol. 112, 665-674.

Ertürk, G., Hedström, M., Tümer, M.A., Denizli, A., Mattiasson, B., 2015. Real-time prostate-specific antigen detection with prostate-specific antigen imprinted capacitive biosensors. Anal. Chim. Acta 891, 120-129. https://doi.org/10.1016/J.ACA. 2015.07.055.

Etayash, H., Jiang, K., Azmi, S., Thundat, T., Kaur, K., 2015. Real-time detection of breast cancer cells using peptide-functionalized microcantilever arrays. Sci. Rep. 5, 13967. https://doi.org/10.1038/srep13967.

Faghri, A., Zhang, Y., Howell, J.R., 2010. Advanced Heat and Mass Transfer. Global Digital Press.

Fan, X., White, I.M., Shopova, S.I., Zhu, H., Suter, J.D., Sun, Y., 2008. Sensitive optical biosensors for unlabeled targets: a review. Anal. Chim. Acta 620, 8-26.

Fernández-Friera, L., Fuster, V., López-Melgar, B., Oliva, B., García-Ruiz, J.M., Mendiguren, J., Bueno, H., Pocock, S., Ibáñez, B., Fernández-Ortiz, A., Sanz, J., 2017. Normal LDL-cholesterol levels are associated with subclinical atherosclerosis in the absence of risk factors. J. Am. Coll. Cardiol. 70, 2979-2991. https://doi.org/10. 1016/j.jacc.2017.10.024.

Fiedler, B.A., Ferguson, M., 2017. Overview of medical device clinical trials. In: Managing Medical Devices Within a Regulatory Framework. Elsevier, pp. 1.

Frascella, F., Petri, C., Ricciardi, S., Napione, L., Munzert, P., Jonas, U., Dostalek, J., Bussolino, F., Fabrizio Pirri, C., Descrovi, E., 2016. Hydrogel-terminated photonic crystal for label-free detection of angiopoietin-1. J. Light. Technol. 34, 3641-3645. https://doi.org/10.1109/JLT.2016.2530876.

Gomes-Filho, S.L.R., Dias, A.C.M.S., Silva, M.M.S., Silva, B.V.M., Dutra, R.F., 2013. A carbon nanotube-based electrochemical immunosensor for cardiac troponin $\mathrm{T}$. Microchem. J. 109, 10-15. https://doi.org/10.1016/j.microc.2012.05.033.

Górecka, E., Jastrzębska, M., 2011. Immobilization techniques and biopolymer carriers. Biotechnol. Food Sci. 75, 65-86.

Grieshaber, D., Mackenzie, R., Vörös, J., Reimhult, E., 2008. Electrochemical biosensorssensor principles and architectures. Sensors 8, 1400.

Guenther, M., 2010. Hydrogel-based piezoresistive biochemical microsensors. SPIE Smart 1-5. https://doi.org/10.1117/12.847373.

Gupta, D., Lis, C.G., 2009. Role of CA125 in predicting ovarian cancer survival - a review of the epidemiological literature. J. Ovarian Res. 2, 1-20. https://doi.org/10.1186/ 1757-2215-2-13.

Gupta, R., Chaudhury, N.K., 2007. Entrapment of biomolecules in sol-gel matrix for applications in biosensors: problems and future prospects. Biosens. Bioelectron. 22, 2387-2399. https://doi.org/10.1016/J.BIOS.2006.12.025.

Halcox, J.P., Roy, C., Tubach, F., Banegas, J.R., Dallongeville, J., De Backer, G., Guallar, E., Sazova, O., Medina, J., Perk, J., Steg, P.G., Rodríguez-Artalejo, F., Borghi, C., 2014. C-reactive protein levels in patients at cardiovascular risk: EURIKA study. BMC Cardiovasc. Disord. 14, 25. https://doi.org/10.1186/1471-2261-14-25.

Halhouli, M., Kieninger, J., Daubinger, P., Yurchenko, O., Urban, G., 2016. Sensitivity and selectivity of porous electrodes in heterogeneous liquid-based catalytic reactions: 3D simulation study. J. Electrochem. Soc. 163, E273-E281. https://doi.org/10.1149/2. 0151610jes.

Halldorsson, S., Lucumi, E., Gómez-Sjöberg, R., Fleming, R.M.T., 2015. Advantages and challenges of microfluidic cell culture in polydimethylsiloxane devices. Biosens. Bioelectron. 63, 218-231. https://doi.org/10.1016/J.BIOS.2014.07.029.

Hammond, J.L., Formisano, N., Estrela, P., Carrara, S., Tkac, J., 2016. Electrochemical biosensors and nanobiosensors. Essays Biochem. 60, 69-80. https://doi.org/10. 1042/EBC20150008.

Han, S.W., Koh, W.-G., 2016. Hydrogel-framed nanofiber matrix integrated with a microfluidic device for fluorescence detection of matrix metalloproteinases-9. Anal. Chem. 88, 6247-6253. https://doi.org/10.1021/acs.analchem.5b04867.

Han, X., Wang, J., Sun, Y., 2017. Circulating tumor DNA as biomarkers for cancer detection. Genom. Proteom. Bioinforma. 15, 59-72. https://doi.org/10.1016/J.GPB 2016.12.004.

Hasan, A., Murunnabi, M., Morshed, M., Paul, A., Polini, A., Kuila, T., Al Harini, M., Lee, Y.K., Jaffa, A.A., 2014. Recent advances in application of biosensors in tissue engineering. BioMed Res. Int. 2014, 307519.

Homaei, A.A., Sariri, R., Vianello, F., Stevanato, R., 2013. Enzyme immobilization: an update. J. Chem. Biol. 6, 185-205. https://doi.org/10.1007/s12154-013-0102-9.

Hou, S., Zhang, A., Su, M., 2016. Nanomaterials for biosensing applications. Nanomaterials 6, 58, https://doi.org/10.3390/nano6040058.

Jang, E., Kim, M., Koh, W.-G., 2015. Ag@SiO2-entrapped hydrogel microarray: a new platform for a metal-enhanced fluorescence-based protein assay. Analyst 140, 3375-3383. https://doi.org/10.1039/c5an00251f.

Jin, R., Dijkstra, P.J., 2010. Hydrogels for tissue engineering applications. In: Biomedical Applications of Hydrogels Handbook. Springer New York, New York, NY, pp. 203-225. https://doi.org/10.1007/978-1-4419-5919-5_11.

Ju, Y.M., Yu, B., Koob, T.J., Moussy, Y., Moussy, F., 2008. A novel porous collagen scaffold around an implantable biosensor for improving biocompatibility. I. In vitro/ in vivo stability of the scaffold and in vitro sensitivity of the glucose sensor with scaffold. J. Biomed. Mater. Res. Part A 87A, 136-146. https://doi.org/10.1002/jbm. a.31756.

Ju, Y.M., Yu, B., West, L., Moussy, Y., Moussy, F., 2009. A novel porous collagen scaffold around an implantable biosensor for improving biocompatibility. II. Long-term in vitro/in vivo sensitivity characteristics of sensors with NDGA- or GA-crosslinked collagen scaffolds. J. Biomed. Mater. Res. Part A 9999A. https://doi.org/10.1002/jbm.a. 32400 .

Jung, Y.K., Kim, J., Mathies, R.A., 2015. Microfluidic linear hydrogel array for multiplexed single nucleotide polymorphism (SNP) detection. Anal. Chem. 87, 3165-3170. https://doi.org/10.1021/ac5048696.

Jung, Y.K., Kim, J., Mathies, R.A., 2016. Microfluidic hydrogel arrays for direct genotyping of clinical samples. Biosens. Bioelectron. 79, 371-378. https://doi.org/10. 1016/J.BIOS.2015.12.068.

Kafizas, A., Godin, R., Durrant, J.R., 2017. Charge carrier dynamics in metal oxide photoelectrodes for water oxidation. Semicond. Semimet. 97, 3-46. https://doi.org/ 10.1016/BS.SEMSEM.2017.02.002.

Kassing, R., 2006. Fabrication of Nanostructures. Springer, Dordrecht, pp. 127-136. https://doi.org/10.1007/1-4020-4594-8_7.

Kazemi, S.H., Ghodsi, E., Abdollahi, S., Nadri, S., 2016. Porous graphene oxide nanostructure as an excellent scaffold for label-free electrochemical biosensor: detection of cardiac troponin I. Mater. Sci. Eng. C 69, 447-452. https://doi.org/10.1016/j.msec. 2016.07.005.

Keyes, M.H., Semersky, F.E., Gray, D.N., 1979. Glucose analysis utilizing immobilized enzymes. Enzym. Microb. Technol. 1, 91-94. https://doi.org/10.1016/01410229(79)90104-2.

Khalaf, M.M., Ibrahimov, H.G., Ismailov, E.H., 2012. Nanostructured materials: importance, synthesis and characterization-a review. Chem. J. 2, 118-125.

Knetsch, M.L.W., Koole, L.H., 2011. New strategies in the development of antimicrobial coatings: the example of increasing usage of silver and silver nanoparticles. Polymers 3, 340-366. https://doi.org/10.3390/polym3010340.

Kress, J., Zanaletti, R., Amour, A., Ladlow, M., Frey, J.G., Bradley, M., 2002. Enzyme accessibility and solid supports: which molecular weight enzymes can be used on solid supports? An investigation using confocal raman microscopy. Chem. - A Eur. J. 8, 3769. https://doi.org/10.1002/1521-3765(20020816)8:16<3769::AIDCHEM3769> 3.0.CO;2-V.

Kunduru, V., Bothara, M., Grosch, J., Sengupta, S., Patra, P.K., Prasad, S., 2010. Nanostructured surfaces for enhanced protein detection toward clinical diagnostics. Nanomed. Nanotechnol. Biol. Med. 6, 642-650. https://doi.org/10.1016/j.nano. 2010.03.002.

Ladgotra, A., Verma, P., Raj, S.S., 2016. Estimation of salivary and serum biomarkers in diabetic and non diabetic patients - a comparative study. J. Clin. Diagn. Res. 10, ZC56-ZC61. https://doi.org/10.7860/JCDR/2016/19135.7995.

Lee, A.G., Arena, C.P., Beebe, D.J., Palecek, S.P., 2010. Development of macroporous poly (ethylene glycol) hydrogel arrays within microfluidic channels. Biomacromolecules 11, 3316-3324. https://doi.org/10.1021/bm100792y.

Lee, D.S., Kim, S.J., Kang, J.H., Hong, S.H., Jeon, E.K., Kim, Y.K., Yoo, I.R., Park, J.G., Jang, H.S., Lee, H.C., Kim, Y.S., 2014. Serum Carcinoembryonic antigen levels and the risk of whole-body metastatic potential in advanced nonsmall cell lung cancer. J. Cancer 5, 663-669. https://doi.org/10.7150/jca.9871.

de Lemos, J.A., Morrow, D.A., Gibson, C.M., Murphy, S.A., Sabatine, M.S., Rifai, N. McCabe, C.H., Antman, E.M., Cannon, C.P., Braunwald, E., 2002. The prognostic value of serum myoglobin in patients with non-ST-segment elevation acute coronary syndromes. Results from the TIMI 11B and TACTICS-TIMI 18 studies. J. Am. Coll. Cardiol. 40, 238-244.

Li, H., Huang, Y., Yu, Y., Li, W., Yin, Y., Li, G., 2015a. Peptide-based method for detection of metastatic transformation in primary tumors of breast cancer. Anal. Chem. 87, 9251-9256. https://doi.org/10.1021/acs.analchem.5b01750. 
Li, L., Wang, Y., Pan, L., Shi, Y., Cheng, W., Shi, Y., Yu, G., 2015b. A nanostructured conductive hydrogels-based biosensor platform for human metabolite detection. Nano Lett. 15, 1146-1151. https://doi.org/10.1021/nl504217p.

Lin, G., Chang, S., Hao, H., Tathireddy, P., Orthner, M., Magda, J., Solzbacher, F., 2010. Osmotic swelling pressure response of smart hydrogels suitable for chronically implantable glucose sensors. Sens. Actuators B Chem. 144, 332-336. https://doi.org/ 10.1016/j.snb.2009.07.054.

Ling, Y., Pong, T., Vassiliou, C.C., Huang, P.L., Cima, M.J., 2011. Implantable magnetic relaxation sensors measure cumulative exposure to cardiac biomarkers. Nat. Biotechnol. 29, 273-277. https://doi.org/10.1038/nbt.1780.

Liu, Z., Ma, H., Sun, H., Gao, R., Liu, H., Wang, X., Xu, P., Xun, L., 2017. Nanoporous goldbased microbial biosensor for direct determination of sulfide. Biosens. Bioelectron. 98, 29-35. https://doi.org/10.1016/J.BIOS.2017.06.037.

Loh, Q.L., Choong, C., 2013. Three-dimensional scaffolds for tissue engineering applications: role of porosity and pore size. Tissue Eng. Part B. Rev. 19, 485-502. https:// doi.org/10.1089/ten.TEB.2012.0437.

Lu, T., Li, Y., 2013. Techniques for fabrication and construction of three-dimensional scaff. Int. J. Nanomed. 8, 337-350.

Mahajan, V.S., Jarolim, P., 2011. How to interpret elevated cardiac troponin levels. Circulation 124, 2350-2354. https://doi.org/10.1161/CIRCULATIONAHA.111. 023697.

Malekzad, H., Sahandi Zangabad, P., Mirshekari, H., Karimi, M., Hamblin, M.R., 2017. Noble metal nanoparticles in biosensors: recent studies and applications. Nanotechnol. Rev. 6, 301-329. https://doi.org/10.1515/ntrev-2016-0014.

Manawi, Y.M., Ihsanullah, Samara, A., Al-Ansari, T., Atieh, M.A., 2018. A review of carbon nanomaterials' synthesis via the chemical vapor deposition (CVD) method. Materials 11. https://doi.org/10.3390/ma11050822.

Martinkova, P., Kostelnik, A., Valek, T., Pohanka, M., 2017. Main streams in the construction of biosensors and their applications. Int. J. Electrochem. Sci. 12, 7386-7403. https://doi.org/10.20964/2017.08.02.

Mazurenko, I., Monsalve, K., Infossi, P., Giudici-Orticoni, M.-T., Topin, F., Mano, N., Lojou, E., 2017. Impact of substrate diffusion and enzyme distribution in 3D-porous electrodes: a combined electrochemical and modelling study of a thermostable H2/ O2 enzymatic fuel cell. Energy Environ. Sci. 10, 1966-1982. https://doi.org/10. 1039/C7EE01830D.

Mazzocchi, R.A., 2016. Medical sensors - defining a pathway to commercialization. ACS Sensors 1, 1167.

Meng, E., 2014. Insight: Implantable medical devices. Lab Chip 14, 3233.

Merceron, T.K., Murphy, S.V., 2015. Hydrogels for 3D bioprinting applications. In: Essentials of 3D Biofabrication and Translation. Elsevier, pp. 249-270. https://doi. org/10.1016/B978-0-12-800972-7.00014-1.

Ming, M., Wei, -Yuan, Senthilkumaran, B., Vigneshvar, S., Sudhakumari, C.C., Prakash, H., 2016. Recent advances in biosensor technology for potential applications-an overview. Front. Bioeng. Biotechnol. 4https://doi.org/10.3389/fbioe.2016.00011. (3389-11).

Mironi-Harpaz, I., Wang, D.Y., Venkatraman, S., Seliktar, D., 2012. Photopolymerization of cell-encapsulating hydrogels: crosslinking efficiency versus cytotoxicity. Acta Biomater. 8, 1838-1848.

Misawa, N., Osaki, T., Takeuchi, S., 2018. Membrane protein-based biosensors. J. R. Soc. Interface 15. https://doi.org/10.1098/rsif.2017.0952.

Misun, P.M., Rothe, J., Schmid, Y.R.F., Hierlemann, A., Frey, O., 2016. Multi-analyte biosensor interface for real-time monitoring of 3D microtissue spheroids in hangingdrop networks. Microsyst. Nanoeng. 2, 16022. https://doi.org/10.1038/micronano. 2016.22.

Mohamad, N.R., Marzuki, N.H.C., Buang, N.A., Huyop, F., Wahab, R.A., 2015. An overview of technologies for immobilization of enzymes and surface analysis techniques for immobilized enzymes. Biotechnol. Biotechnol. Equip. 29, 205-220. https://doi. org/10.1080/13102818.2015.1008192.

Mordente, A., Meucci, E., Martorana, G.E., Silvestrini, A., 2015. Cancer biomarkers discovery and validation: state of the art, problems and future perspectives. In: Advances in Cancer Biomarkers. Springer, pp. 9-26. https://doi.org/10.1007/978-94-0177215-0 2

Muskovich, M., Bettinger, C.J., 2012. Biomaterials-based electronics: polymers and interfaces for biology and medicine. Adv. Healthc. Mater. 1, 248-266. https://doi.org/ 10.1002/adhm.201200071.

Nikitin, M.P., Orlov, A.V., Znoyko, S.L., Bragina, V.A., Gorshkov, B.G., Ksenevich, T.I., Cherkasov, V.R., Nikitin, P.I., 2018. Multiplex biosensing with highly sensitive magnetic nanoparticle quantification method. J. Magn. Magn. Mater. 459, 260-264. https://doi.org/10.1016/J.JMMM.2017.10.078.

Onuki, Y., Upkar, M.P., Papadimitrakopoulos, F., Burgess, D.J., 2008. A review of the biocompatibility of implantable devices: current challenges to overcome foreign body response. J. Diabetes Sci. Technol. 2.

Patlolla, A., Knighten, B., Tchounwou, P., 2010. Multi-walled carbon nanotubes induce cytotoxicity, genotoxicity and apoptosis in normal human dermal fibroblast cells. Ethn. Dis. 20.

Pedrosa, V.A., Yan, J., Simonian, A.L., Revzin, A., 2011. Micropatterned nanocomposite hydrogels for biosensing applications. Electroanalysis 23, 1142-1149. https://doi. org/10.1002/elan.201000654.

Piao, Y., Han, D.J., Azad, M.R., Park, M., Seo, T.S., 2015. Enzyme incorporated microfluidic device for in-situ glucose detection in water-in-air microdroplets. Biosens. Bioelectron. 65, 220-225. https://doi.org/10.1016/J.BIOS.2014.10.032.

Pina, S., Rebelo, R., Correlo, V.M., Oliveira, J.M., Reis, R.L., 2018. Bioceramics for osteochondral tissue engineering and regeneration. In: Oliveira, J.M., Pina, S., Reis, R.L., San Roman, J. (Eds.), Osteochondral Tissue Engineering: Nanotechnology, Scaffolding-Related Developments and Translation. 1058. Springer International Publishing, pp. 53.
Poruk, K.E., Gay, D.Z., Brown, K., Mulvihill, J.D., Boucher, K.M., Scaife, C.L., Firpo, M.A., Mulvihill, S.J., 2013. The clinical utility of CA 19-9 in pancreatic adenocarcinoma: diagnostic and prognostic updates. Curr. Mol. Med. 13, 340-351.

Profusa, Inc [WWW Document], n.d. URL 〈https://profusa.com/〉 (Accessed 23 July 2018).

Qu, F., Zhang, Y., Rasooly, A., Yang, M., 2014. Electrochemical biosensing platform using hydrogel prepared from ferrocene modified amino acid as highly efficient immobilization matrix. Anal. Chem. 86, 973-976. https://doi.org/10.1021/ac403478z.

Quintela, I., Corte, M.D., Allende, M.T., Vazquez, J., Rodríguez, J.C., Bongera, M., Lamelas, M., Gonzalez, L.O., Vega, A., García-Muñiz, J.L., Astudillo, A., Vizoso, F. 2005. Expression and prognostic value of EGFR in invasive breast cancer. Oncol. Rep. 14, 1655-1663.

Rai, M., Yadav, A., Gade, A., 2009. Silver nanoparticles as a new generation of antimicrobials. Biotechnol. Adv. 27, 76-83. https://doi.org/10.1016/j.biotechadv.2008. 09.002 .

Rajput, N., 2015. Methods of preparation of nanoparticles-a review. Int. J. Adv. Eng. Technol. 7, 1806-1811.

Rogers, K.R., 2000. Principles of affinity-based biosensors. Affin. Biosens. 109 Mol. Biotechnol. 14.

Rogers, M.L., Boutelle, M.G., 2013. Real-time clinical monitoring of biomolecules. Annu. Rev. Anal. Chem. 6, 427-453. https://doi.org/10.1146/annurev.anchem.111808. 073648.

Rong, Q., Han, H., Feng, F., Ma, Z., 2015. Network nanostructured polypyrrole hydrogel/ $\mathrm{Au}$ composites as enhanced electrochemical biosensing platform. Sci. Rep. 5, 11440 https://doi.org/10.1038/srep11440.

Sabury, S., Kazemi, S.H., Sharif, F., 2015. Graphene-gold nanoparticle composite: application as a good scaffold for construction of glucose oxidase biosensor. Mater. Sci. Eng. C 49, 297-304. https://doi.org/10.1016/j.msec.2015.01.018.

Saerens, D., Huang, L., Bonroy, K., Muyldermans, S., 2008. Antibody fragments as probe in biosensor development. Sensors 8, 4669-4686. https://doi.org/10.3390/ s8084669.

Sasso, L.A., Aran, K., Guan, Y., Ündar, A., Zahn, J.D., 2013. Continuous monitoring of inflammation biomarkers during simulated cardiopulmonary bypass using a microfluidic immunoassay device-a pilot study. Artif. Organs 37, E9-E17. https://doi.org/ 10.1111/aor.12021.

Shan, J., Ma, Z., 2017. A review on amperometric immunoassays for tumor markers based on the use of hybrid materials consisting of conducting polymers and noble metal nanomaterials. Microchim. Acta 184, 969-979. https://doi.org/10.1007/s00604017-2146-y.

Singh, M., Holzinger, M., Biloivan, O., Cosnier, S., 2013. 3D-nanostructured scaffold electrodes based on single-walled carbon nanotubes and nanodiamonds for high performance biosensors. Carbon N. Y. 61, 349-356.

Slaughter, G., Sunday, J., 2014. Fabrication of enzymatic glucose hydrogel biosensor based on hydrothermally grown ZnO nanoclusters. IEEE Sens. J. 14, 1573-1576. https://doi.org/10.1109/JSEN.2014.2298359.

Son, K.J., Gheibi, P., Stybayeva, G., Rahimian, A., Revzin, A., 2017. Detecting cell-secreted growth factors in microfluidic devices using bead-based biosensors. Microsyst. Nanoeng. 3, 17025. https://doi.org/10.1038/micronano.2017.25.

Srinivasan, M., Blackburn, C., Mohamed, M., Sivagami, A.V., Blum, J., 2015. Literaturebased discovery of salivary biomarkers for type 2 diabetes mellitus. Biomark. Insights 10, 39-45. https://doi.org/10.4137/BMI.S22177.

Srisuk, P., Berti, F.V., da Silva, L.P., Marques, A.P., Reis, R.L., Correlo, V.M., 2018. Electroactive gellan gum/polyaniline spongy-like hydrogels. ACS Biomater. Sci. Eng. 4https://doi.org/10.1021/acsbiomaterials.7b00917. (17779-1787)

Sun, K., Tang, Y., Li, Q., Yin, S., Qin, W., Yu, J., Chiu, D.T., Liu, Y., Yuan, Z., Zhang, X., $\mathrm{Wu}, \mathrm{C} ., 2$ 2016. In vivo dynamic monitoring of small molecules with implantable polymer-dot transducer. ACS Nano 10, 6769-6781. https://doi.org/10.1021/ acsnano.6b02386.

Tanaka, H., Hanasaki, M., Isojima, T., Takeuchi, H., Shiroya, T., Kawaguchi, H., 2009. Enhancement of sensitivity of SPR protein microarray using a novel 3D protein immobilization. Colloids Surf. B Biointerfaces 70, 259-265. https://doi.org/10.1016/J. COLSURFB. 2008.12.037.

Taplin, C.E., Barker, J.M., 2008. Autoantibodies in type 1 diabetes. Autoimmunity 41 , 11-18. https://doi.org/10.1080/08916930701619169.

Thapa, A., Soares, A.C., Soares, J.C., Awan, I.T., Volpati, D., Melendez, M.E., Fregnani, J.H.T.G., Carvalho, A.L., Oliveira, O.N., 2017. Carbon nanotube matrix for highly sensitive biosensors to detect pancreatic cancer biomarker CA19-9. ACS Appl. Mater. Interfaces 9, 25878-25886. https://doi.org/10.1021/acsami.7b07384.

Tierney, S., Falch, B.M.H., Hjelme, D.R., Stokke, B.T., 2009. Determination of glucose levels using a functionalized hydrogel-optical fiber biosensor: toward continuous monitoring of blood glucose in vivo. Anal. Chem. 81, 3630-3636. https://doi.org/10. 1021/ac900019k.

Tiwari, J.N., Tiwari, R.N., Kim, K.S., 2012. Zero-dimensional, one-dimensional, two-dimensional and three-dimensional nanostructured materials for advanced electrochemical energy devices. Prog. Mater. Sci. 57, 724-803. https://doi.org/10.1016/j. pmatsci.2011.08.003.

Urban, G.A., Weiss, T., 2009. Hydrogels for biosensors. Hydrogel Sens. Actuators 197-220. https://doi.org/10.1007/978-3-540-75645-3_6.

Uzun, L., Turner, A.P.F., 2016. Molecularly-imprinted polymer sensors: realising their potential. Biosens. Bioelectron. 76, 131-144.

Vasapollo, G., Sole, R., Del, Mergola, L., Lazzoi, M.R., Scardino, A., Scorrano, S., Mele, G., 2011. Molecularly imprinted polymers: present and future prospective. Int. J. Mol. Sci. 12, 5908-5945, https://doi.org/10.3390/ijms12095908.

Viswanathan, S., Rani, C., Ribeiro, S., Delerue-Matos, C., 2012. Molecular imprinted nanoelectrodes for ultra sensitive detection of ovarian cancer marker. Biosens. Bioelectron. 33, 179-183. https://doi.org/10.1016/j.bios.2011.12.049. 
Wang, C., Feng, B., 2015. Research progress on site-oriented and three-dimensional immobilization of protein. Mol. Biol. 49, 1-20. https://doi.org/10.1134/ S0026893315010173.

Wang, J., Wang, Y., Chen, H., Xu, H., Wang, W., Bai, L., 2018a. Sensitive and simultaneous detection of tumor markers assisted by novel functional polymer brush/Au nanoparticles composite. Sens. Actuators B Chem. 258, 998-1007. https://doi.org/ 10.1016/J.SNB.2017.11.186.

Wang, S., Zhang, X., Huang, J., Chen, J., 2018b. High-performance non-enzymatic catalysts based on 3D hierarchical hollow porous $\mathrm{Co} 3 \mathrm{O} 4$ nanododecahedras in situ decorated on carbon nanotubes for glucose detection and biofuel cell application. Anal. Bioanal. Chem. 410, 2019-2029. https://doi.org/10.1007/s00216-018-0875-3.

Welch, N.G., Scoble, J.A., Muir, B.W., Pigram, P.J., 2017. Orientation and characterization of immobilized antibodies for improved immunoassays (Review). Cit. Biointerphases 12, 2-301. https://doi.org/10.1116/1.4978435.

WHO, 2017. [WWW Document]. WHO. URL 〈http://www.who.int/mediacentre/ factsheets/fs310/en/ $>$ (Accessed 9 January 2017)

Widayani, Yanti, Wungu, T.D.K., Suprijadi, 2017. Preliminary study of molecularly imprinted polymer-based potentiometric sensor for glucose. Procedia Eng. 170, 84-87.

Yang, P., Chen, N., Yang, D., Crane, J., Yang, S., Wang, H., Dong, R., Yi, X., Xie, L., Jing, G., Cai, J., Wang, Z., 2017. The ratio of serum Angiopoietin-1 to Angiopoietin-2 in patients with cervical cancer is a valuable diagnostic and prognostic biomarker. PeerJ
5, e3387. https://doi.org/10.7717/peerj.3387.

Yang, T.-Y., Fang, C.-Y., Chen, J.-S., Po, H.L., Chou, L.-P., Chiang, C.-Y., Ueng, K.-C., 2015. Association of serum uric acid with cardiovascular disease in taiwanese patients with primary hypertension. Acta Cardiol. Sin. 31, 42-51. https://doi.org/10. 6515/ACS20140120A.

Ye, J., Baumgaertel, A.C., Wang, Y.M., Biener, J., Biener, M.M., 2015. Structural optimization of 3D porous electrodes for high-rate performance lithium ion batteries. ACS Nano 9, 2194-2202. https://doi.org/10.1021/nn505490u.

Yu, Q., Wang, Q., Li, B., Lin, Q., Duan, Y., 2015. Technological development of antibody immobilization for optical immunoassays: progress and prospects. Crit. Rev. Anal. Chem. 45, 62-75. https://doi.org/10.1080/10408347.2014.881249.

Zanghelini, F., Frías, I.A.M., Rêgo, M.J.B.M., Pitta, M.G.R., Sacilloti, M., Oliveira, M.D.L., Andrade, C.A.S., 2017. Biosensing breast cancer cells based on a three-dimensional TIO 2 nanomembrane transducer. Biosens. Bioelectron. 92, 313-320. https://doi. org/10.1016/j.bios.2016.11.006.

Zhang, S., Wright, G., Yang, Y., 2000. Materials and techniques for electrochemical biosensor design and construction. Biosens. Bioelectron. 15, 273-282.

Zhu, C., Yang, G., Li, H., Du, D., Lin, Y., 2015. Electrochemical sensors and biosensors based on nanomaterials and nanostructures. Anal. Chem. 87, 230-249. https://doi. org/10.1021/ac5039863. 